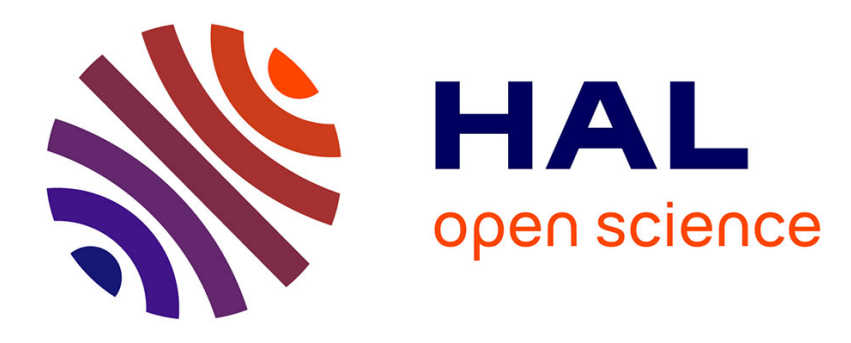

\title{
Exergy-Based Formulation for Aircraft Aeropropulsive Performance Assessment: Theoretical Development
}

\author{
A. Arntz, O. Atinault, A. Merlen
}

\section{To cite this version:}

A. Arntz, O. Atinault, A. Merlen. Exergy-Based Formulation for Aircraft Aeropropulsive Performance Assessment: Theoretical Development. AIAA Journal, 2014, pp.1 - 38. 10.2514/1.J053467 . hal01096423

\section{HAL Id: hal-01096423 \\ https://hal.science/hal-01096423}

Submitted on 17 Dec 2014

HAL is a multi-disciplinary open access archive for the deposit and dissemination of scientific research documents, whether they are published or not. The documents may come from teaching and research institutions in France or abroad, or from public or private research centers.
L'archive ouverte pluridisciplinaire HAL, est destinée au dépôt et à la diffusion de documents scientifiques de niveau recherche, publiés ou non, émanant des établissements d'enseignement et de recherche français ou étrangers, des laboratoires publics ou privés. 


\title{
Exergy-based Formulation for Aircraft Aeropropulsive
}

\section{Performance Assessment: Theoretical Development}

\author{
Aurélien Arntz ${ }^{1}$, Olivier Atinault ${ }^{2}$, and Alain Merlen ${ }^{3}$ \\ ONERA - The French Aerospace Lab, France
}

\begin{abstract}
Aircraft have evolved into extremely complex systems that require adapted methodologies and tools for an efficient design process. A theoretical formulation based on exergy management is proposed for assessing the aeropropulsive performance of future aircraft configurations. It consists in the combination of a momentum balance and a fluid flow analysis involving on the first and second laws of thermodynamics. The exergy supplied by the propulsion system and its partial destruction within the control volume is associated with the aircraft mechanical equilibrium. Characterization of the recoverable mechanical and thermal outflows is made along with the identification of the irreversible phenomena that destroy their work potential. Restriction of the formulation to unpowered configurations yields connections to some well-known far-field drag expressions and shows that their underlying theory can be related to exergy considerations. As the exergy balance does not rely on the distinction of thrust and drag, it is especially suitable for the performance evaluation of highly integrated aeropropulsive concepts like boundary layer ingestion.
\end{abstract}

\footnotetext{
${ }^{1}$ PhD Student, Department of Applied Aerodynamics, aurelien.arntz@onera.fr.

2 Research Engineer, Department of Applied Aerodynamics, olivier.atinault@onera.fr.

${ }^{3}$ Chief Scientist of the Fluid Mechanics and Energetics Branch, alain.merlen@ onera.fr.
} 


\section{Nomenclature}

$\dot{\mathcal{A}}_{q} \quad=$ rate of heat anergy supplied by conduction $\left(\mathrm{J} . \mathrm{s}^{-1}\right)$

$\dot{\mathcal{A}}_{\nabla T} \quad=$ rate of anergy generation by thermal mixing $\left(\mathrm{J} . \mathrm{s}^{-1}\right)$

$\dot{\mathcal{A}}_{\phi} \quad=$ rate of anergy generation by viscous dissipation $\left(\mathrm{J} . \mathrm{s}^{-1}\right)$

$\dot{\mathcal{A}}_{\text {tot }} \quad=$ rate of total anergy generation $\left(\mathrm{J} . \mathrm{s}^{-1}\right),=\dot{\mathcal{A}}_{\phi}+\dot{\mathcal{A}}_{\nabla T}+\dot{\mathcal{A}}_{w}$

$\dot{\mathcal{A}}_{w} \quad=$ rate of anergy generation by shock waves $\left(\mathrm{J} . \mathrm{s}^{-1}\right)$

$D \quad=$ aerodynamic drag $(\mathrm{N})$

$e \quad=$ mass specific internal energy $\left(\mathrm{J} \cdot \mathrm{kg}^{-1}\right)$

$E S F C=$ exergy specific fuel consumption

$\dot{E}_{u} \quad=$ streamwise kinetic energy deposition rate $\left(\mathrm{J} . \mathrm{s}^{-1}\right)$

$\dot{E}_{p} \quad=$ boundary pressure-work rate $\left(\mathrm{J} . \mathrm{s}^{-1}\right)$

$\dot{E}_{\phi} \quad=$ rate of thermal energy generation by viscous dissipation $\left(\mathrm{J} . \mathrm{s}^{-1}\right)$

$\dot{E}_{q} \quad=$ rate of heat energy supplied by conduction $\left(\mathrm{J} . \mathrm{s}^{-1}\right)$

$\dot{E}_{t h} \quad=$ rate of thermal energy outflow $\left(\mathrm{J} . \mathrm{s}^{-1}\right)$

$\dot{E}_{v} \quad=$ transverse kinetic energy deposition rate $\left(\mathrm{J} . \mathrm{s}^{-1}\right)$

$\dot{E}_{W} \quad=$ surroundings-work rate $\left(\mathrm{J} . \mathrm{s}^{-1}\right)$

$\varepsilon \quad=$ mass specific flow exergy $\left(\mathrm{J}^{\mathrm{kgg}}{ }^{-1}\right)$

$\dot{\mathcal{E}}_{m} \quad=$ rate of mechanical exergy outflow $\left(\mathrm{J} . \mathrm{s}^{-1}\right),=\dot{E}_{u}+\dot{E}_{v}+\dot{E}_{p}$

$\dot{\mathcal{E}}_{\text {prop }}=$ rate of exergy supplied by the propulsion system $\left(\mathrm{J} . \mathrm{s}^{-1}\right)$

$\dot{\mathcal{E}}_{\text {prop }, m}=$ rate of mechanical exergy supplied by the propulsion system $\left(\mathrm{J} . \mathrm{s}^{-1}\right)$

$\dot{\mathcal{E}}_{\text {prop }, t h}=$ rate of thermal exergy supplied by the propulsion system (J.s $\left.{ }^{-1}\right)$

$\dot{\mathcal{E}}_{\phi} \quad=$ rate of thermal exergy generation by viscous dissipation $\left(\mathrm{J} . \mathrm{s}^{-1}\right)$

$\dot{\mathcal{E}}_{q} \quad=$ rate of heat exergy supplied by conduction $\left(\mathrm{J} . \mathrm{s}^{-1}\right)$

$\dot{\mathcal{E}}_{t h} \quad=$ rate of thermal exergy outflow $\left(\mathrm{J} . \mathrm{s}^{-1}\right)$

$F_{\text {prop }}=$ momentum change across the propulsive surface $(\mathrm{N})$

$F_{x} \quad=$ streamwise resultant force acting on the vehicle $(\mathrm{N})$ 
$\Delta h_{f}=$ mass specific fuel heating value $\left({\mathrm{J} . \mathrm{kg}^{-1}}^{-1}\right)$

$\Gamma \quad=$ weight specific aircraft energy height $(\mathrm{m})$

$h_{i} \quad=$ mass specific total enthalpy $\left(\mathrm{J}_{\mathrm{kg}} \mathrm{kg}^{-1}\right)$

$k=$ medium thermal conductivity $\left(\mathrm{W} \cdot \mathrm{m}^{-1} \cdot \mathrm{K}^{-1}\right)$

$\dot{m}_{f}=$ fuel mass flow $\left(\mathrm{kg} \cdot \mathrm{s}^{-1}\right)$

$\mathbf{n}=$ unit normal vector

$\Phi=$ dissipation rate per unit volume $\left(\mathrm{J} \cdot \mathrm{s}^{-1} \cdot \mathrm{m}^{-3}\right)$

$\psi_{\varepsilon} \quad=$ overall exergy efficiency

$\psi_{i} \quad=$ engine intrinsic exergy efficiency

$\psi_{m}=$ mechanical exergy efficiency

$\psi_{p} \quad=$ propulsive efficiency

$\mathbf{q}=$ heat flux by conduction $\left(\mathrm{J} . \mathrm{s}^{-1}\right)$

$\mathrm{s} \quad=$ mass specific entropy $\left(\mathrm{J} \cdot \mathrm{K}^{-1} \cdot \mathrm{kg}^{-1}\right)$

$\overline{\bar{\tau}} \quad=$ viscous stress tensor $(\mathrm{N})$

$\mathbf{V}=$ fluid velocity vector $\left(\mathrm{m} \cdot \mathrm{s}^{-1}\right),=\left(V_{\infty}+u\right) \mathbf{x}, v \mathbf{y}, w \mathbf{z}$

$W \quad=$ aircraft weight $(\mathrm{N})$

\section{Subscripts}

$A=$ aircraft surface

$B$ = aircraft body surface

$\infty=$ quantity at freestream conditions

$O=$ outer boundary

$P=$ propulsion system surface

\section{Operator}

$=$ time rate of change

$\delta()=$ quantity relative to freestream $=()-()_{\infty}$ 


\section{Introduction}

\section{Problem Statement}

A century after the first commercial flight made in January 1914, the air transportation industry has known a fantastic growth, especially since the early 50's where air traffic has doubled every 15-20 years. Most forecasts predict a continuing 5\%-per annum growth, at least for the two coming decades. Such a growth has raised environmental concern and driven international institutions to set ambitious objectives of performance. Among these objectives emerged a dramatic reduction in both fuel combustion and noise/pollutant emission. It is likely that the continuous improvement of the conventional tube-and-wings configuration will not be sufficient to satisfy these new constraints. One of the most promising configurations is the blended-wing body (BWB) featuring a boundary layer ingestion (BLI) propulsion [? ? ]. However, this configuration is rather unconventional and current tools and methodology become inapplicable. Especially the conventional drag-thrust bookkeepings, used to separate the task of the airframe designer from the one of the propulsion engineer, becomes excessively ambiguous for such highly integrated propulsion systems [? ? ? ]. This lack of methodology prevents an accurate estimation of the benefit of a complete advanced configuration and represents the main motivation for deriving the present performance prediction formulation.

For transonic (to supersonic) commercial aircraft, this challenge has been taken up by Drela [? ] who proposed a formulation based on a mechanical energy analysis which does not rely on the expression of forces (thrust/drag). The formulation allows the identification of all external flow phenomena usually of interest for the airframe designer: shock waves, lift-induced vortices and wake dissipation. It has notably been applied for a computational assessment of the boundary layer ingesting nacelle design of the D8 aircraft [?] and used for wind-tunnel experiments [?].

Drela's formulation provides an efficient tool for mechanical performance estimation but does not allow for aircraft thermal management. This thermal aspect has been identified as being an area for major gain in military aircraft performance [? ] and is also expected to play an increasing role for commercial aircraft [?] as a greater number of electromechanical actuation systems are anticipated to create thermal loads that can be challenging to manage [? ]. A key example of advanced configuration is the N3-X concept which decouples the power producing parts of the system from the thrust producing parts of the system thereby 
allowing each to be optimized for its task [? ? ]. It utilizes superconducting electrically driven, distributed low-pressure-ratio fans with power provided by two remote superconducting electric generators based on a conventional turbofan core engine design [? ]. This shift from existing aircraft concepts adds a great deal of complexity to the system through various energy exchanges and it stands to reason, that, as a greater number of energy forms is expected to be dealt with for future aircraft, the need for a common currency to assess the performance of various sub-systems (and their interactions) may be reinforced.

The formulation presented here aims at providing a more global tool for the design of such complex configurations by taking into account the thermal energy in addition to the mechanical energy. These two forms represent the total energy of the fluid and appear equivalent by virtue of the first law of thermodynamics. However, in the perspective of producing work, it is clear that while the mechanical energy is completely convertible into mechanical work, the thermal energy is not. According to the second law of thermodynamics, only a portion of it could be ideally converted into mechanical work via a Carnot cycle of efficiency lower than unity. The mechanical energy can thus be considered a higher quality form of energy than the thermal energy. Even for thermal energy itself, there can be a ranking because, the hotter the gas, the higher the thermodynamic efficiency. The distinction of the quality and level of an energy form is only made possible by the introduction of the second law of thermodynamics. The combination of both laws enables an exergy analysis which starts by splitting any form of energy in two parts: a first part (theoretically) fully convertible into mechanical work and a second part that is (theoretically) impossible to be converted into mechanical work. Following the work of Rant [? ] to establish an international terminology, it seems that the words exergy and anergy are well suited to represent these two parts. Other names can be found in the literature for exergy, especially availability made popular by Keenan [? ]. As exergy is the part of energy that has economic value [? ], it is the only part worth managing carefully.

\section{Exergy Approach to Aerospace Vehicle Design}

The exergy analysis has been successfully applied to the design of ground-based systems such as fuel cells, heat exchangers, cryogenics, chemical processes, solar power, distillation and desalination [? ? ? ]. In the aerospace community, a growing interest in the exergy analysis can be observed since the very early 2000's [? ? ]. The approach consists in considering that it is the usage and loss of thermodynamic 
work potential that drives virtually every aspect of a vehicle's environmental and economic performance [? ]. Far from being an obscure scientific notion, the exergy analysis yields a fundamental definition of loss in an engineering perspective: exergy destruction, or equivalently anergy generation, by irreversible phenomena. As a consequence, it tells the designer how much improvement is possible and how close the actual system is to ideal [? ]. Pioneer Moorhouse expanded exergy methods to the design of a complete flight vehicle by defining mission requirements as an exergy problem cascading down to each component in the same framework [? ]. The ability to perform exergy accounting in smaller and smaller subsystems makes it possible to draw a map of how the destruction of exergy is distributed over the system of interest which is a real advantage in the search for improving efficiency of a system [without a priori knowledge], because it tells us from the start how to allocate engineering efforts and ressources [? ]. As the ultimate figure of merit for loss in any vehicle regardless of type or construction is cost [? ], the exergy analysis can be combined with costing considerations to form a thermoeconomic analysis. This approach provides information not available through conventional energy analysis and economic evaluations but crucial to the design of a cost effective system [? ]. The exergy analysis appears as a system-level approach that could improve the energy management of highly advanced aerospace vehicles [? ] as providing a metric whereby thermal and chemical energies are directly comparable to mechanical and electrical energies [?].

Most applications have focused on propulsion systems [? ? ? ] following the pioneering work of Clarke and Horlock [? ] to analyse a propulsive device in the light of the combined first and second laws. They identified a consistent framework within which losses can be compared within machines, between machines of different types for the same job and with perfection represented by the concept of the completely reversible machine. s the operating conditions, which play a major role in the performance of the system, may vary throughout the flight of an air vehicle, the possibility of the exergy analysis to formally take into account variations makes it possible to optimize the system for its entire mission [? ? ? ? ? ] by accurately guiding improvement efforts [?].

There has been significantly less interest on external flows and most noticeable is the work of Roth [? ] to extend the exergy approach to define aerodynamic loss as the reduction in total vehicle work potential due to irreversible fluid-dynamic interactions between the vehicle and the atmosphere. On that account, aerodynamic loss is directly comparable to thermodynamic loss in the engine and part of the system global 
cost accounting [? ? ]. Also note that an analytical model for the design and optimization of an aerospace vehicle was developed by Riggins et al. [? ? ? ] who brought together the equations of motion and an exergy balance for the analysis of hypersonic vehicles.

The present article follows these recent efforts and introduces the theoretical developments of a new formulation in the perspective of CFD-RANS applications. Note that the formulation has already been numerically implemented and successfully applied to various airframe cases [? ] as well as for the investigation of the BLI concept [?].

\section{Fluid Flow Analysis}

\section{A. Control Volume}

The fluid flow analysis is carried out in a continuous volume $\mathcal{V}$ delimited by the aircraft surface $\mathcal{S}_{A}$, a surface $\mathcal{S}_{w}$ excluding any discontinuous shock wave, and an outer boundary $\mathcal{S}_{O}$. All surfaces are closed and a vector pointing outwards of the volume and locally normal to the surface is noted $\mathbf{n}$, as illustrated in Fig. ??. The control volume delimits the system under study which is said to be thermodynamically open

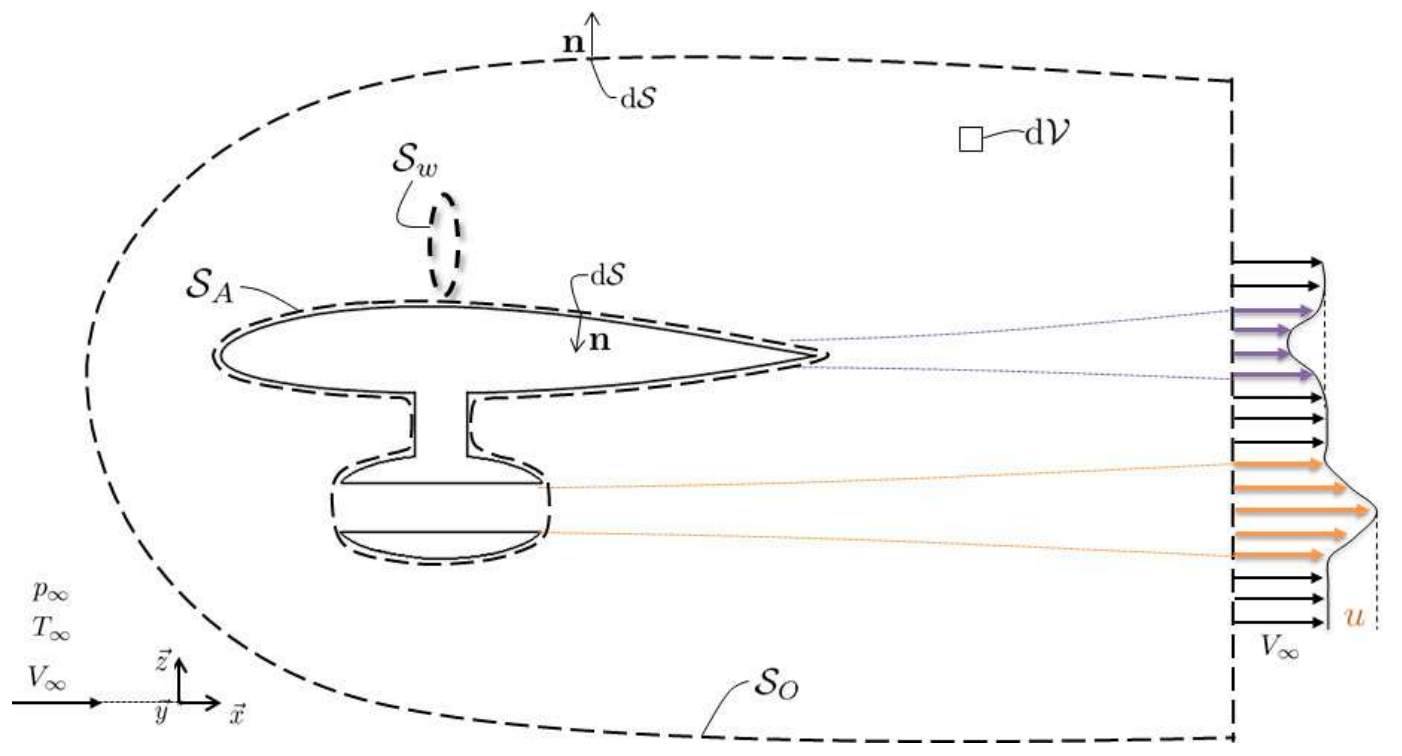

Fig. $12 \mathrm{D}$ cutaway view of 3D control volume surrounding the aircraft.

as it can exchange mass, work, and heat with the surrounding fluid across its boundaries. The atmosphere is considered as a thermal and mechanical reservoir, which means that we neglect the influence of the process being studied on the (much larger) surroundings. The analysis is made in the aircraft reference frame, the 
control volume is therefore fixed and the air is flowing in and out of it.

Note that, although the form of the outer boundary is arbitrary, it is sometimes found advantageous to choose a downstream plane perpendicular to the freestream velocity and to push the remaining lateral and upstream surfaces as far as possible from the aircraft. This has the practical advantage of assuming freestream conditions on all surfaces but the downstream plane. Although, this assumption is often made for drag prediction formulations and could be used to slightly simplify the mathematical derivation (and the physical interpretation) [? ], this approach is not adopted for the following derivation in order to be as general as possible.

Extensive use of the divergence theorem will be made in the derivation:

$$
\int_{\mathcal{V}} \nabla \cdot() \mathrm{d} \mathcal{V}=\int_{\mathcal{S}_{A}}() \cdot \mathbf{n} \mathrm{d} \mathcal{S}+\int_{\mathcal{S}_{w}}() \cdot \mathbf{n} \mathrm{d} \mathcal{S}+\int_{\mathcal{S}_{O}}() \cdot \mathbf{n} \mathrm{d} \mathcal{S}
$$

where () is any continuous tensor quantity.

\section{B. Mean Steady Flows and Boussinesq's Hypothesis}

The objective is to develop a post-processing code for the preliminary design of future commercial transport aircraft for which RANS numerical solutions provide valuable performance indications at a moderate computational cost. In the perspective of developing such a numerical tool, the following derivation is restricted to compressible and mean steady flows. Application of the Reynolds- and Favre-averaging procedures to the Navier-Stokes equations introduces two major unknowns: the turbulent shear stress and the turbulent heat flux. As described in [? ], these turbulent quantities can be expressed as functions of mean quantities for mean steady flows.

First, the Boussinesq's hypothesis considers that the turbulent shear stress is locally related linearly to

the mean rate of strain $\overline{\bar{S}}$; the proportionality factor being an eddy viscosity noted $\mu_{t}$. As a consequence, neglecting the turbulent kinetic energy contribution, the effective (viscous + turbulent) stress tensor can be expressed as $\overline{\bar{\tau}}_{e f f}=\left(\mu+\mu_{t}\right) \overline{\bar{S}}$. Similarly, the effective dissipation rate due to viscous dissipation and turbulence is given by $\Phi_{\text {eff }}=\left(\overline{\bar{\tau}}_{\text {eff }} \cdot \nabla\right) \cdot \mathbf{V}$. In the following the adjective effective is replaced by viscous for convenience.

Secondly, in a direct analogy to the Boussinesq's hypothesis, the effective heat flux by conduction, introducing the Fourier law, can be written as $\mathbf{q}_{\text {eff }}=-k_{\text {eff }} \nabla \mathbf{T}$. The effective thermal conductivity is 
expressed as $k_{e f f}=c_{p}\left(\mu / \operatorname{Pr}+\mu_{t} / P r_{t}\right)$, where the turbulent Prandtl number is usually chosen as a constant value of $\operatorname{Pr}_{t}=0.9$ for air. As a consequence, the turbulent heat flux only requires a value for the eddy viscosity.

For brevity, the notations for Reynolds- and Favre-averaged are disregarded in the following derivation as well as the notation eff.

\section{Mass Conservation}

The mass conservation principle is applied to the control volume to provide a relation used to manipulate and simplify other subsequent integral relations. The steady-state Reynolds-averaged mass relation in local form is as follows:

$$
\nabla \cdot(\rho \mathbf{V})=0
$$

Integrating within the control volume $\mathcal{V}$ and using the divergence theorem yields:

$$
\dot{m}_{A}=\dot{m}_{O}
$$

where

$$
\begin{gathered}
\dot{m}_{A}:=-\int_{\mathcal{S}_{A}} \rho(\mathbf{V} \cdot \mathbf{n}) \mathrm{d} \mathcal{S}=\dot{m}_{f} \simeq 0 \\
\dot{m}_{O}:=\int_{\mathcal{S}_{O}} \rho(\mathbf{V} \cdot \mathbf{n}) \mathrm{d} \mathcal{S}=\dot{m}_{f} \simeq 0
\end{gathered}
$$

The fuel mass flow $\dot{m}_{f}$ will be assumed negligible compared to the air mass flow considered within the control volume, so that both integrals are negligible. Thus, we have, for any scalar quantity convected by the flow like the total enthalpy $h_{i}$ :

$$
\int_{\mathcal{S}} \rho h_{i}(\mathbf{V} \cdot \mathbf{n}) \mathrm{d} \mathcal{S}=\int_{\mathcal{S}} \rho\left(h_{i}-h_{i_{\infty}}\right)(\mathbf{V} \cdot \mathbf{n}) \mathrm{d} \mathcal{S}=\int_{\mathcal{S}} \rho \delta h_{i}(\mathbf{V} \cdot \mathbf{n}) \mathrm{d} \mathcal{S}
$$

where $\mathcal{S}$ is any closed surface of the analysis. This property is used to build the exergy balance in section ??.

\section{Momentum Relation}

The momentum relation is used to obtain a far-field expression for the power developed by the net force acting on the aircraft. The steady-state Reynolds-averaged momentum relation in divergence form is as 
follows:

$$
\nabla \cdot(\rho \mathbf{V} \otimes \mathbf{V})=-\nabla \mathbf{p}+\nabla \cdot \overline{\bar{\tau}}
$$

Again, integrating within the control volume and invoking the divergence theorem yields the two following momentum fluxes:

$$
\mathbf{F}_{\mathbf{A}}=\mathbf{F}_{\mathbf{O}}
$$

where the two terms represent the net force acting on the aircraft, $\mathbf{F}_{\mathbf{A}}$ being a near-field integral while $\mathbf{F}_{\mathbf{O}}$ is a far-field expression:

$$
\begin{gathered}
\mathbf{F}_{\mathbf{A}}:=\int_{\mathcal{S}_{A}}\left[\rho \mathbf{V}(\mathbf{V} \cdot \mathbf{n})+\left(p-p_{\infty}\right) \mathbf{n}-(\overline{\bar{\tau}} \cdot \mathbf{n})\right] \mathrm{d} \mathcal{S} \\
\mathbf{F}_{\mathbf{O}}:=-\int_{\mathcal{S}_{O}}\left[\rho \mathbf{V}(\mathbf{V} \cdot \mathbf{n})+\left(p-p_{\infty}\right) \mathbf{n}\right] \mathrm{d} \mathcal{S}
\end{gathered}
$$

Note that the viscous force has been dropped from (??) as it is rapidly negligible compared to the other forces involved at distances greater than one body length downstream of the configuration [? ? ]. As shown in appendix A, the rate of work done by this force[? ] is $W \dot{\Gamma}$ :

$$
W \dot{\Gamma}=-\mathbf{F}_{\mathbf{O}} \cdot \mathbf{V}_{\infty}=\int_{\mathcal{S}_{O}}\left[\rho u V_{\infty}(\mathbf{V} \cdot \mathbf{n})+\left(p-p_{\infty}\right)\left(\mathbf{V}_{\infty} \cdot \mathbf{n}\right)\right] \mathrm{d} \mathcal{S}
$$

where $\Gamma$ is the weight specific aircraft energy height, i.e. the sum of the aircraft potential and kinetic energies [? ]. This expression represents the power of the resultant force and there is no need (and no possibility) to distinguish thrust from drag. The following subsections aims at building a first and second law balance which will identify this far-field expression of $W \dot{\Gamma}$ in section ??.

\section{E. First Law of Thermodynamics}

First, the total enthalpy is defined as the sum of the internal energy, the flow energy and the kinetic energy and its rate of change can therefore be written as:

$$
\nabla \cdot\left(\rho \delta h_{i} \mathbf{V}\right)=\nabla \cdot(\rho \delta e \mathbf{V})+p_{\infty} \nabla \cdot \mathbf{V}+\nabla \cdot\left(p-p_{\infty}\right) \mathbf{V}+\nabla \cdot\left(\rho \frac{V^{2}}{2} \mathbf{V}\right)
$$

where we have added and subtracted $p_{\infty} \nabla \cdot \mathbf{V}$ for further purposes. 
Secondly, according to the first law of thermodynamics, the steady-state Reynolds-averaged total enthalpy equation is as follows:

$$
\nabla \cdot\left(\rho \delta h_{i} \mathbf{V}\right)=\nabla \cdot(\overline{\bar{\tau}} \cdot \mathbf{V})-\nabla \cdot \mathbf{q}
$$

where $\mathbf{q}$ is the heat flux by conduction.

\section{F. Second Law of Thermodynamics}

The Gibbs relation provides a relation between entropy and internal energy. In time-averaged form it reads:

$$
T \nabla \mathrm{s}=\nabla e+p \nabla \frac{1}{\rho}
$$

Inserting the steady-state expression of the internal energy variation

$$
\nabla \cdot(\rho \delta e \mathbf{V})=-p \nabla \cdot \mathbf{V}+(\overline{\bar{\tau}} \cdot \nabla) \cdot \mathbf{V}-\nabla \cdot \mathbf{q}
$$

into the Gibbs relation (??) yields the following entropy relation:

$$
\nabla \cdot(\rho \delta \mathrm{s} \mathbf{V})=\frac{1}{T}(\overline{\bar{\tau}} \cdot \nabla) \cdot \mathbf{V}-\frac{1}{T} \nabla \cdot \mathbf{q}
$$

Introducing the Fourier law and the dissipation rate $\Phi$, one gets, multiplying Eq. (??) by the reference temperature $T_{\infty}$ :

$$
T_{\infty} \nabla \cdot(\rho \delta \mathrm{s} \mathbf{V})=\frac{T_{\infty}}{T} \Phi+\frac{T_{\infty}}{T^{2}} k(\nabla \mathbf{T})^{2}-\nabla \cdot \frac{T_{\infty}}{T} \mathbf{q}
$$

which corresponds to Moore and Moore eddy viscosity model for mean entropy production [? ]. It requires the assumption that the rate of production of turbulence kinetic energy equals the rate of its dissipation and that the rate of production of temperature fluctuations equals their rate of dissipation. Although higher fidelity models exist [? ], this simple model is preferred in the present work as it only relies on the eddy viscosity and on quantities of the mean flow. This final relation is used in the following section to form the exergy balance. 


\section{Exergy Balance}

\section{A. Definitions \& Terminology}

Exergy is a thermodynamic property describing the maximum theoretical work that can be obtained from a substance in taking it from a given temperature and pressure/velocity to a state of thermal and mechanical equilibrium with the environment[? ]. Exergy is a property of both the system and the reference environment, the latter being here taken as the atmosphere at the altitude of flight and whose properties are noted with the subscript $\infty$. As mentioned in the introduction, in the perspective of producing work, we can say that:

$$
\text { Energy }=\text { Exergy }+ \text { Anergy } \quad \Longleftrightarrow \quad \text { Total }=\text { Useful }+ \text { Useless }
$$

which can be translated mathematically for an open system as:

$$
\varepsilon=\left(h_{i}-h_{i_{\infty}}\right)-T_{\infty}\left(\mathrm{s}-\mathrm{s}_{\infty}\right)=\delta h_{i}-T_{\infty} \delta \mathrm{s}
$$

where we have neglected the gravitational potential energy of the specific flow exergy $\varepsilon$ [? ? ]. We also assume that the mixture of air and fuel behaves as a perfect gas. We are interested in calculating the timeaveraged change in exergy which can be written locally as:

$$
\nabla \cdot(\rho \varepsilon \mathbf{V})=\nabla \cdot\left(\rho \delta h_{i} \mathbf{V}\right)-T_{\infty} \nabla \cdot(\rho \delta \mathbf{s} \mathbf{V})
$$

The two previous paragraphs provide an expression for the two right-hand side terms of Eq. (??) that are used in the following subsection to form the exergy balance.

\section{B. General Exergy-based Formulation}

To simplify the derivation we neglect the heat transfer across the outer boundary as well as viscous terms[?]. From the divergence theorem, the integration within the control volume of Eq. (??) yields:

$$
-\int_{\mathcal{S}_{A}} \rho \varepsilon(\mathbf{V} \cdot \mathbf{n}) \mathrm{d} \mathcal{S}=-\int_{\mathcal{V}} \nabla \cdot \rho \varepsilon \mathbf{V} \mathrm{d} \mathcal{V}+\int_{\mathcal{S}_{O}} \rho \varepsilon(\mathbf{V} \cdot \mathbf{n}) \mathrm{d} \mathcal{S}+\int_{\mathcal{S}_{w}} \rho \varepsilon(\mathbf{V} \cdot \mathbf{n}) \mathrm{d} \mathcal{S}
$$

which, inserting Eqs. (??, ?? \& ??) and using Eqs. (?? \& ??), yields the following exergy balance:

$$
\dot{\mathcal{E}}_{\text {prop }}+\dot{\mathcal{E}}_{q}=W \dot{\Gamma}+\dot{\mathcal{E}}_{m}+\dot{\mathcal{E}}_{t h}+\dot{\mathcal{A}}_{t o t}
$$

where the rate of exergy outflow is decomposed into $W \dot{\Gamma}+\dot{\mathcal{E}}_{m}+\dot{\mathcal{E}}_{t h}$ and $\dot{\mathcal{A}}_{t o t}$ represents to total anergy generated within the control volume, $\dot{\mathcal{A}}_{t o t}:=\dot{\mathcal{A}}_{\phi}+\dot{\mathcal{A}}_{\nabla T}+\dot{\mathcal{A}}_{w}$. Left-hand side terms represent exergy 
sources supplied (either by convection or conduction) while right-hand side terms represent exergy outflows and sinks, except for $W \dot{\Gamma}$ which is a (reversible) accumulation/restitution of exergy as shown in paragraph ??. All terms are defined hereafter and sketched in Fig. ??.

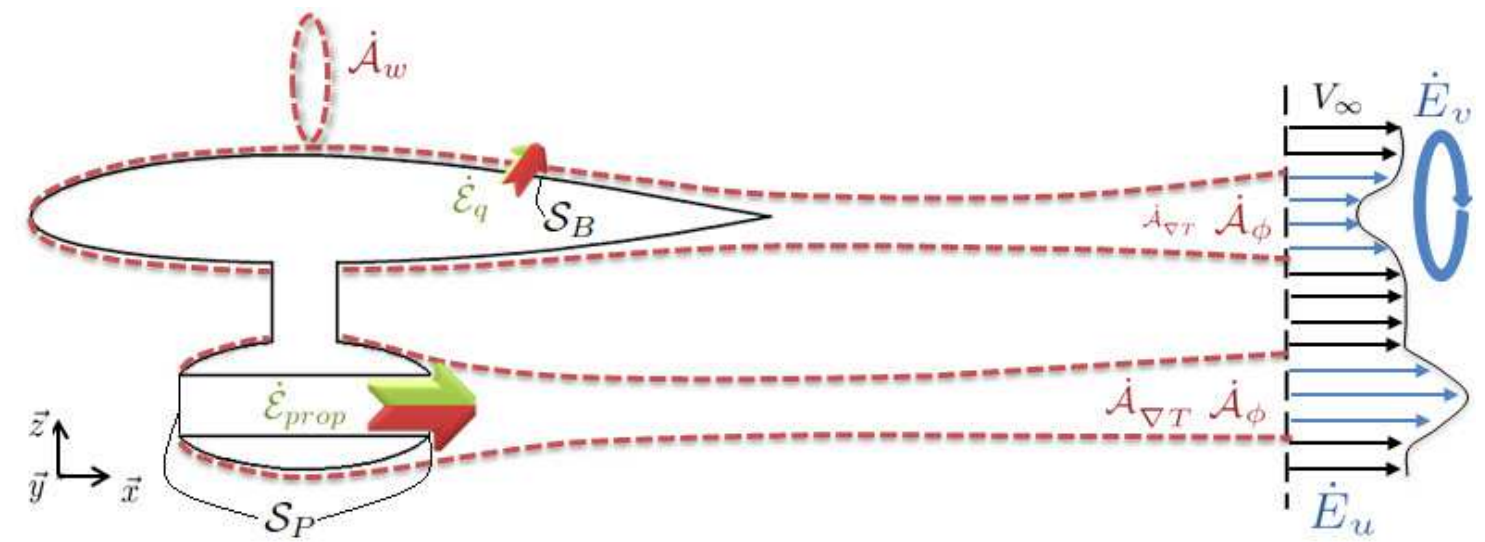

Fig. 2 Main terms of the exergy balance in the flow field. Green is associated with exergy, red to anergy and blue to energy. $\dot{\mathcal{E}}_{t h}$ and $\dot{E}_{p}$ are not shown.

The aircraft surface has been split in two surfaces: one solid body surface $\mathcal{S}_{B}$ which may be nonadiabatic and one permeable propulsive surface $\mathcal{S}_{P}$ on which $\mathbf{V} \cdot \mathbf{n} \neq 0$. Note that: $\mathcal{S}_{A}=\mathcal{S}_{B} \bigcup \mathcal{S}_{P}$.

$\dot{\mathcal{E}}_{\text {prop }}:$ the rate of exergy supplied by the propulsion system

$$
\dot{\mathcal{E}}_{\text {prop }}:=\underbrace{\int_{\mathcal{S}_{P}}-\rho \delta h_{i}(\mathbf{V} \cdot \mathbf{n}) \mathrm{d} \mathcal{S}}_{\dot{\mathcal{H}}_{i}}-\underbrace{T_{\infty} \int_{\mathcal{S}_{P}}-\rho \delta \mathrm{s}(\mathbf{V} \cdot \mathbf{n}) \mathrm{d} \mathcal{S}}_{\dot{\mathcal{A}}_{\text {prop }}}
$$

Both terms are non-zero only on permeable surfaces of the aircraft $\mathcal{S}_{A}$ considered to be only the one associated with the propulsion system $\mathcal{S}_{P}$. The first term is the total power supplied to the flow while the second term represents all unavoidable thermodynamic cycle inefficiencies and aerodynamic losses that have occurred within the propulsion system delimited by the surface $\mathcal{S}_{P}[?]$. The combination of both forms the exergy delivered by the propulsion system to the fluid. A decomposition of this term is provided in subsection ??

Note that $\mathbf{n}$ points into the propulsor, so that the nozzle has $\mathbf{V} \cdot \mathbf{n}<0$, and $\dot{\mathcal{E}}_{\text {prop }}>0$ for a propulsive system with net thrust, as expected. For convenience, $\dot{\mathcal{E}}_{\text {prop }}$ will be referred to as propulsive exergy. 
$\dot{\mathcal{E}}_{q}:$ the rate of heat exergy supplied by conduction

$$
\dot{\mathcal{E}}_{q}:=\underbrace{\int_{\mathcal{S}_{B}}-(\mathbf{q} \cdot \mathbf{n}) \mathrm{d} \mathcal{S}}_{\dot{E}_{q}}-\underbrace{\int_{\mathcal{S}_{B}}-\frac{T_{\infty}}{T}(\mathbf{q} \cdot \mathbf{n}) \mathrm{d} \mathcal{S}}_{\dot{\mathcal{A}}_{q}}
$$

The terms are non-zero only on non-adiabatic surfaces of the body that are noted $\mathcal{S}_{B}$. The first term is the amount of energy of the heat transferred by conduction while the second term is the associated anergy. The combination of both terms yields the exergy supplied to the fluid by conduction. In concrete terms, heat is being supplied to the flow and a potential for being converted to work exists: the higher the temperature (with reference to atmospheric conditions), the greater the work potential. A heat transfer at temperature $T=T_{\infty}$ has no practical value: its thermal exergy is zero [? ]. Note that if $T<T_{\infty}$, the term $\dot{\mathcal{E}}_{q}$ is still positive meaning that there is a potential to improve a Carnot cycle efficiency by using the cold surface as a heat sink.

These terms could notably be used to evaluate systems like heat exchangers. If adiabatic surfaces are considered, which is generally assumed for most civil aircraft, these terms are simply zero.

$\dot{\mathcal{E}}_{m}:$ the rate of mechanical exergy outflow

$$
\dot{\mathcal{E}}_{m}:=\underbrace{\int_{\mathcal{S}_{O}} \frac{1}{2} \rho u^{2}(\mathbf{V} \cdot \mathbf{n}) \mathrm{d} \mathcal{S}}_{\dot{E}_{u}}+\underbrace{\int_{\mathcal{S}_{O}} \frac{1}{2} \rho\left(v^{2}+w^{2}\right)(\mathbf{V} \cdot \mathbf{n}) \mathrm{d} \mathcal{S}}_{\dot{E}_{v}}+\underbrace{\int_{\mathcal{S}_{O}}\left(p-p_{\infty}\right)\left[\left(\mathbf{V}-\mathbf{V}_{\infty}\right) \cdot \mathbf{n}\right] \mathrm{d} \mathcal{S}}_{\dot{E}_{p}}
$$

$\dot{E}_{u}$ is the streamwise kinetic energy deposition rate and is associaed to jet/wake while $\dot{E}_{v}$ is the transverse kinetic energy deposition rates and is mainly associated with lift-induced vortices. $\dot{E}_{p}$ is a boundary pressure-work rate associated with the these flow phenomena. All three terms being linked by velocity/pressure exchanges, they are gathered to form the rate of net mechanical energy outflow.

These terms being of mechanical nature, they represent exergy, and thus indicate a potential for improvement as discussed in the next section. However, for most aerospace applications, the exergy associated with these flow phenomena is not recovered and, for that reason, represent an exergy loss.

$\dot{\mathcal{E}}_{t h}:$ the rate of thermal exergy outflow

$$
\dot{\mathcal{E}}_{t h}:=\underbrace{\int_{\mathcal{S}_{O}} \rho \delta e(\mathbf{V} \cdot \mathbf{n}) \mathrm{d} \mathcal{S}}_{\dot{E}_{t h}}+\underbrace{\int_{\mathcal{S}_{O}} p_{\infty}(\mathbf{V} \cdot \mathbf{n}) \mathrm{d} \mathcal{S}}_{\dot{E}_{W}}-\underbrace{T_{\infty} \int_{\mathcal{S}_{O}} \rho \delta \mathrm{s}(\mathbf{V} \cdot \mathbf{n}) \mathrm{d} \mathcal{S}}_{\dot{\mathcal{A}}}
$$


Assuming a perfect gas, internal energy is solely proportional to temperature $\left(e=c_{v} T\right)$, so that the first term is the rate of thermal energy outflow. The second term is usually referred to as the rate of (isobaric) surroundings work [? ? ] and is a non available work that the system performs, or receives, due to its interaction with the reference atmospheric pressure field at $p_{\infty}[?]$. The last term is the outflow rate of anergy. The combination of the three terms yields the maximum amount of work that is theoretically extractable from the thermal energy. To extract this work one should consider a reversible Carnot cycle using the thermal energy as a source (or sink).

For most aerospace applications, this term is not recovered and therefore represents an exergy loss.

$\dot{\mathcal{A}}_{\phi}:$ the rate of anergy generation by viscous dissipation

$$
\dot{\mathcal{A}}_{\phi}:=\int_{\mathcal{V}} \frac{T_{\infty}}{T} \Phi \mathrm{d} \mathcal{V}
$$

$\dot{\mathcal{A}}_{\phi}$ is linked to viscous dissipation and turbulence which transform any difference in kinetic energy into thermal energy. For that reason, these phenomena act towards reducing the mechanical exergy to zero by building a new homogeneous field of velocity (and pressure), in which the system and its surroundings are back in mechanical equilibrium.

This term is positive and hence will always increase anergy; it indicates an irreversible process that destroy exergy. Even if at high Reynolds numbers the dissipation is mainly associated with turbulence rather than viscous stresses [? ], for convenience, $\dot{\mathcal{A}}_{\phi}$ will be referred to as viscous anergy.

$\dot{\mathcal{A}}_{\nabla T}:$ the rate of anergy generation by thermal mixing

$$
\dot{\mathcal{A}}_{\nabla T}:=\int_{\mathcal{V}} \frac{T_{\infty}}{T^{2}} k(\nabla T)^{2} \mathrm{~d} \mathcal{V}
$$

$\dot{\mathcal{A}}_{\nabla T}$ is linked to thermal mixing which reduces the difference in temperature and therefore reduces the thermal exergy to zero. It acts towards building a new homogeneous field of temperature in which the system and its surroundings are back in thermal equilibrium. The thermal mixing is a function of the square of the temperature gradients so that avoiding strong temperatures variations would reduce this term.

This term is positive and hence will always increase anergy; it indicates an irreversible process that destroy exergy. For convenience, $\dot{\mathcal{A}}_{\nabla T}$ will be referred to as thermal anergy. 
$\dot{\mathcal{A}}_{w}:$ the rate of anergy generation by shock waves

$$
\dot{\mathcal{A}}_{w}:=-T_{\infty} \int_{\mathcal{S}_{w}} \rho \delta \mathrm{s}(\mathbf{V} \cdot \mathbf{n}) \mathrm{d} \mathcal{S}
$$

This definition is the most straightforward way of calculating the anergy associated with discontinuous shock waves. This phenomenon converts mechanical energy into thermal energy; this process being irreversible, it is an exergy destruction. For convenience, $\dot{\mathcal{A}}_{w}$ will be referred to as wave anergy.

The exergy balance Eq. (??) is valid for compressible viscous and mean steady flows with energy supply from a propulsion system and via heat transfer at the surface of the aircraft. Very few assumptions have been made only to simplify the derivation which does not require small perturbations assumption or Taylor series. It is worth noticing that although the balance holds for any location of the downstream plane, numerically speaking, it is found highly advantageous to place it as near as possible to the body in order to minimize the effect of spurious dissipation on lift-induced vortex decay [? ? ].

For adiabatic surfaces, the exergy relation (??) can be described as follows: the exergy supplied by the propulsive system has been used to effectively apply a net force that drives the vehicle through the air. Exergy has been supplied in excess to that requirement in the form of streamwise and transverse kinetic energy and a boundary pressure work. This loss of mechanical exergy is associated with wakes/jets and liftinduced vortices and will be dissipated by viscous dissipation and shock waves. Additionally, work could be extracted from the thermal energy flowing out of the control volume. If not valued, this work potential will be destroyed by thermal mixing. To conclude, in a vehicle performance perspective, the design that wastes and destroys the less exergy is the most efficient one because a correspondingly lower propulsive exergy will be required to compensate.

\section{Outflows Characterization and Recovery}

In an engineering point of view, the definition of loss as entropy generation helps the designer visualizing the flow in a different perspective:

- $\dot{E}_{u}$ is positive regardless the sign of the perturbation axial velocity $u$ which is positive in a jet and negative in a wake. From streamwise momentum considerations, the wake of an aircraft is seen as a deficit, and as a consequence, is considered a loss. The exergy analysis tells us that it actually 
represents a potential for improvement. The concept that takes advantage from having generated a wake is called wake ingestion, or, by extension, boundary layer ingestion [? ]. The idea is to place the propulsive system downstream of the configuration so as to ingest its wake, and to ideally reenergize it by just the right amount to match freestream conditions, leaving no wake/jet: $\dot{E}_{u}=0$. Such arrangement enables the recovery of the exergy present in the wake [? ]. From Eq. (??), one can also see that it would result in a correspondingly lower exergy to be supplied by the propulsion system.

- $\dot{E}_{v}$ represents (mainly) the kinetic energy associated with lift-induced vortices. Traditional far-field momentum-based analysis conclude that these vortices generate drag and are thus generally viewed as losses. In the exergy/anergy framework, the formation of the vortex is not considered as a direct loss[? ]. The exergy approach offers a different flow perspective in considering the vortex formation as a potential for work extraction which can be valued through different processes such as formation flight [? ] or wingtip turbines [? ? ]. Again, there would be a correspondingly lower exergy to be supplied by the propulsion system.

- $\dot{\mathcal{E}}_{t h}$ could actually be termed thermocompressible exergy as it can be decomposed into a (pure) thermal exergy at constant volume and a thermal exergy associated with volume change, as shown in appendix B. The dominance of one aspect over the other one will depend on the application under study. For example, for a realistic powered aircraft flying at low speed, it is likely that the thermal exergy at constant volume part would be the largest contributor to the overall thermocompressible exergy due to high-temperature jet exhaust while for an unpowered configuration at transonic regime, the thermal exergy associated with volume may be the largest contributor. For convenience, thermal exergy is kept to designate $\dot{\mathcal{E}}_{t h}$.

It is also shown in appendix B that the thermal exergy is positive no matter if $T$ is higher than $T_{\infty}$ or not. This is because a temperature lower than reference conditions can be used as a temperature sink to improve the efficiency of an ideal Carnot cycle while a temperature higher than $T_{\infty}$ could be used as a source.

Although it does not necessarily take an exergy analysis to recognize that the flow downstream a configuration could offer improvements, by redefining the loss as entropy generation, the exergy/anergy framework 
provides a consistent approach to evaluate any recoverable potential. As soon as the local state is not in equilibrium with the reference conditions, exergy is available, and the more it deviates from equilibrium, the greater the work potential [? ]. For unconventional configurations for which we have little knowledge, this approach would make any (mechanical or thermal) waste clearly visible.

\section{Propulsion Analysis}

\section{Exergy Efficiencies}

The scope of this analysis is voluntarily large so as being able to deal with any kind of propulsive device. As $\dot{\mathcal{E}}_{\text {prop }}$ represents the potential to produce propulsive power, one can first introduce an engine intrinsic exergy efficiency $\psi_{i}$ :

$$
\psi_{i}:=\frac{\dot{\mathcal{E}}_{\text {prop }}}{\dot{\mathcal{H}}_{i}}=1-\frac{\dot{\mathcal{\mathcal { A }}}_{\text {prop }}}{\dot{\mathcal{H}}_{i}}
$$

where $\dot{\mathcal{H}}_{i}$ is the rate of total energy supplied to the flow while $\dot{\mathcal{A}}_{\text {prop }}$ represents all anergy generation that has occured within the propulsive device delimited by the surface boundary $\mathcal{S}_{P}$. The primary source of energy for $\dot{\mathcal{H}}_{i}$ could be of any form (electrical or chemical). For example, for fuel-based propulsion, the total power supplied to the flow is equal to the rate of energy released by combustion [? ] $\dot{\mathcal{H}}_{i}=\dot{m}_{f} \Delta h_{f}$ where $\Delta h_{f}$ is the fuel higher heating value, roughly[? ] equal to the (standard) chemical exergy stored in the fuel [? ].

It is crucial to note that, if the propulsion system is modeled in the computation, use of the divergence theorem could be made to decompose $\dot{\mathcal{A}}_{\text {prop }}$ into viscous anergy, thermal anergy, wave anergy and heat transfer (by conduction) anergy. Moreover, this decomposition could be obtained for each component of the complete propulsive device.

The exergy delivered by the propulsion can be decomposed into a mechanical exergy and a thermal exergy (if any):

$$
\dot{\mathcal{E}}_{\text {prop }}=\dot{\mathcal{E}}_{\text {prop }, m}+\dot{\mathcal{E}}_{\text {prop }, t h}
$$

where

$$
\begin{gathered}
\dot{\mathcal{E}}_{\text {prop }, m}=\int_{\mathcal{S}_{P}}\left[\left(p-p_{\infty}\right)+\frac{1}{2} \rho\left(V^{2}-V_{\infty}^{2}\right)\right](\mathbf{V} \cdot \hat{\mathbf{n}}) \mathrm{d} \mathcal{S} \\
\dot{\mathcal{E}}_{\text {prop }, t h}=\int_{\mathcal{S}_{P}}\left[\rho\left(\delta e-T_{\infty} \delta \mathbf{s}\right)+p_{\infty}\right](\mathbf{V} \cdot \hat{\mathbf{n}}) \mathrm{d} \mathcal{S}
\end{gathered}
$$


where we introduced $\hat{\mathbf{n}}=-\mathbf{n}$ for writing convenience. In terms of propulsion, only the mechanical part of the total exergy is in a usable form. One can therefore introduce a mechanical efficiency as:

$$
\psi_{m}:=\frac{\dot{\mathcal{E}}_{\text {prop }, m}}{\dot{\mathcal{E}}_{\text {prop }}}
$$

For a given exergy supplied to the flow, as the thermal exergy is reduced, the mechanical efficiency is increased. The mechanical exergy can then be expanded into:

$$
\begin{aligned}
\dot{\mathcal{E}}_{\text {prop }, m}= & \underbrace{\int_{\mathcal{S}_{P}}\left[\rho \mathbf{V}(\mathbf{V} \cdot \hat{\mathbf{n}})+\left(p-p_{\infty}\right) \hat{\mathbf{n}}\right] \mathrm{d} \mathcal{S}}_{F_{\text {prop }}} \cdot \mathbf{V}_{\infty}+\underbrace{\int_{\mathcal{S}_{P}} \frac{1}{2} \rho u^{2}(\mathbf{V} \cdot \hat{\mathbf{n}}) \mathrm{d} \mathcal{S}}_{\text {Axial Kinetic Power }} \\
& +\underbrace{\int_{\mathcal{S}_{P}} \frac{1}{2} \rho\left(v^{2}+w^{2}\right)(\mathbf{V} \cdot \hat{\mathbf{n}}) \mathrm{d} \mathcal{S}}_{\text {Transverse Kinetic Power }}+\underbrace{\int_{\mathcal{S}_{P}}\left(p-p_{\infty}\right)\left[\left(\mathbf{V}-\mathbf{V}_{\infty}\right) \cdot \hat{\mathbf{n}}\right] \mathrm{d} \mathcal{S}}_{\text {Pressure-Work Rate }}
\end{aligned}
$$

where the first term is the dot product of the momentum change across the surface $\mathcal{S}_{P}$ and of the flight velocity $\mathbf{V}_{\infty}$. Whether or not the force $F_{\text {prop }}$ should be considered an (intrinsic/gross) thrust is left at the discretion of airframe and propulsion designers, with the understanding that the surface $\mathcal{S}_{P}$ has to be defined. The difficulty lies in the fact that the definition of $\mathcal{S}_{P}$ is not unique, especially for highly integrated propulsion devices, as illustrated in Fig. ??, where it could be any combination of inlet/outlet surfaces.

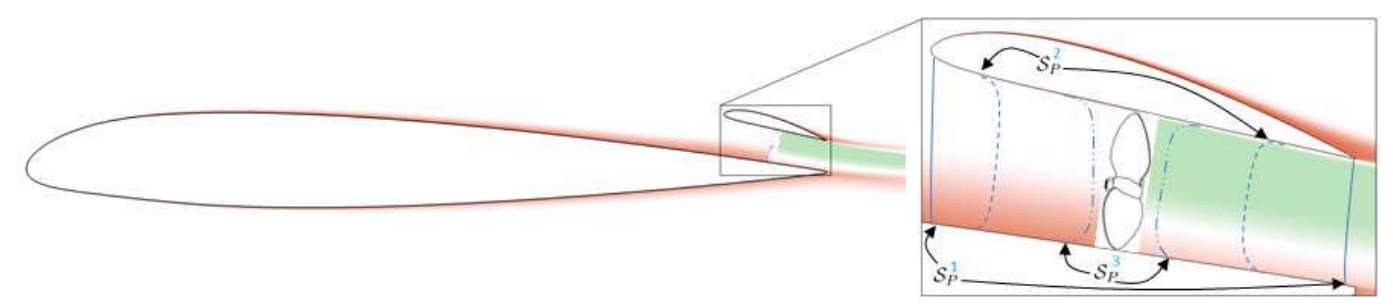

Fig. 3 Possible choices for the propulsive surface definition for a highly integrated propulsion system.

In fact, the force $F_{\text {prop }}$ is only identical to thrust under the conditions that the surface is carefully chosen so at to satisfy a given drag/thrust bookkeeping, which always involves some kind of arbitrariness [?] and must be adapted to any particular situation [? ].

The propulsive efficiency $\psi_{p}$ indicates the portion of mechanical energy that has been effectively converted into gross propulsive power:

$$
\psi_{p}:=\frac{\mathbb{F}_{\text {prop }} V_{\infty}}{\dot{\mathcal{E}}_{\text {prop }, m}}
$$

This efficiency is capable of assessing under/over-expanded nozzles for which $p \neq p_{\infty}$. Note that if the surface $S_{P}$ is taken such that we can assume $p=p_{\infty}$, then $\psi_{p}$ reduces to its standard expression as ratio of 
thrust power to the kinetic power [? ]. One can finally introduce an overall exergy efficiency $\psi_{\varepsilon}$ :

$$
\psi_{\varepsilon}:=\frac{\mathbb{F}_{\text {prop }} V_{\infty}}{\dot{\mathcal{H}}_{i}}=\psi_{i} \times \psi_{m} \times \psi_{p}
$$

These efficiencies are applicable to any type of propulsion system and should therefore be well-suited for technology comparisons.

\section{Refinement for Jet Propulsion}

The basic exergy analysis proposed above yields absolute loss relative to the maximum work allowed by the second law of thermodynamics whereby any departure from a Carnot cycle appears as a loss. It has been found that application of this approach to jet propulsion devices concludes that the largest exergy losses are due to nonequilibrium combustion and exhaust heat and kinetic energy [? ? ]. However, it appears that optimization of a thrust-producing device to produce maximum exergy output may lead to less-than-optimal result if the objective is to produce thrust for propulsion [? ]. Two additional figures of merit derived from exergy considerations are therefore worth noticing: Gas Horsepower and Thrust Work Potential [? ]. The former only considers mechanical equilibrium and thus concludes that nonequilibrium combustion and heat losses no longer appear as losses; the only inefficiency being associated with exhaust kinetic energy. Thrust work potential is defined as the thrust work that would be obtained in expanding a flow at a given temperature and pressure to ambiant such that the thrust work obtained is equal to the thrust produced multiplied by the flight velocity of the aircraft [? ]. As a consequence, exhaust residual kinetic energy is not counted as being available for propulsive purposes and therefore not accounted as loss, unlike exergy and GHP. Also, relationships between classic measures of component efficiencies to modern measures of work potential have been proposed, the former having the disadvantage of not being directly comparable to one another [? ].

\section{E. Further Specifications of the Formulation}

\section{Thermal Exergy and Mechanical Exergy Relations}

Relation (??) somehow hides the fact that the viscous dissipation is a process that represents a loss in mechanical exergy $\dot{E}_{\phi}$, but a gain in thermal exergy $\dot{\mathcal{E}}_{\phi}$ at temperature $T$, the net destruction of exergy being 
$\dot{\mathcal{A}}_{\phi}:$

$$
\dot{\mathcal{A}}_{\phi}=\int_{\mathcal{V}} \frac{T_{\infty}}{T} \Phi \mathrm{d} \mathcal{V}=\underbrace{\int_{\mathcal{V}} \Phi \mathrm{d} \mathcal{V}}_{\dot{E}_{\phi}}-\underbrace{\int_{\mathcal{V}}\left(1-\frac{T_{\infty}}{T}\right) \Phi \mathrm{d} \mathcal{V}}_{\dot{\mathcal{E}}_{\phi}}
$$

As expected, exergy is the portion that could be converted into mechanical work via an ideal Carnot cycle of efficiency $1-T_{\infty} / T$. The thermal exergy associated with viscous dissipation is highest when the temperature at which the process takes place is highest, with reference to the ambient conditions. Equivalently, the loss (anergy generation) associated with viscous dissipation is lowest when the temperature at which it occurs is highest [? ]. This statement is true for any irreversibility meaning that anergy generation at high temperature is less crucial than at lower temperature [?].

From Eq. (??), it is possible to re-write the main exergy balance Eq. (??) as:

$$
\dot{\mathcal{E}}_{\text {prop }}+\dot{\mathcal{E}}_{q}+\dot{\mathcal{E}}_{\phi}=W \dot{\Gamma}+\dot{\mathcal{E}}_{m}+\dot{\mathcal{E}}_{t h}+\dot{E}_{\phi}+\dot{\mathcal{A}}_{\nabla T}+\dot{\mathcal{A}}_{w}
$$

where $\dot{E}_{\phi}$ appears as a sink of mechanical exergy while $\dot{\mathcal{E}}_{\phi}$ represents a source of thermal exergy (thus placed left-hand side). In the light of these developments, Eq. (??) can be graphically represented as in Fig. ?? for an aircraft in equilibrium.

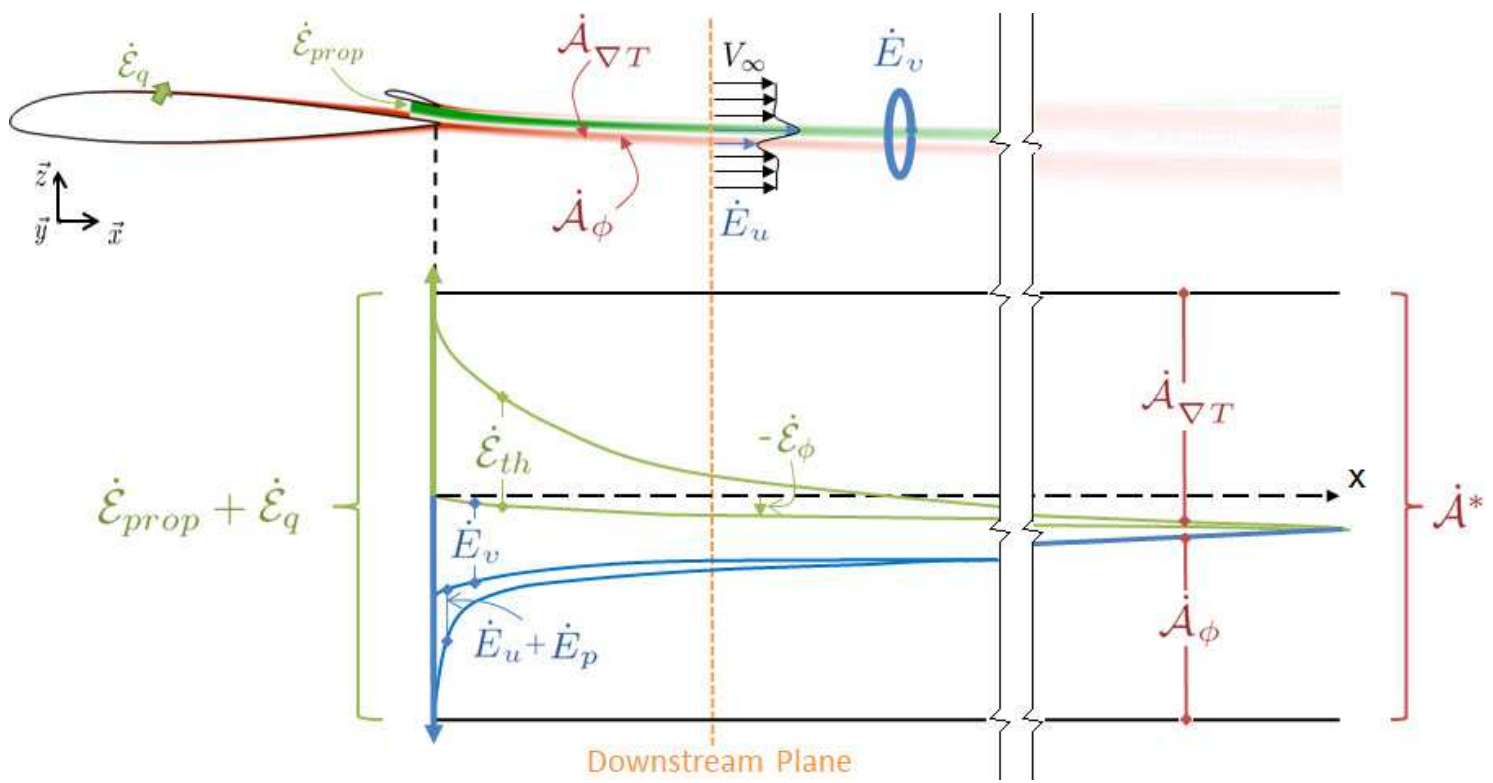

Fig. 4 Variation in the exergy balance terms of Eq. (??) versus position of the downstream plane for an aircraft in mechanical equilibrium. $\dot{\mathcal{A}}_{w}$ is not shown.

The transverse kinetic energy is dissipated much later than the streamwise component. Both kinetic 
energies dissipation provides the thermal exergy $\dot{\mathcal{E}}_{\phi}$ which is itself dissipated by thermal mixing. Thermal anergy $\dot{\mathcal{A}}_{\nabla T}$ is mostly associated with jet exhaust thermal exergy dissipation while viscous anergy $\dot{\mathcal{A}}_{\phi}$ is linked to both wake and jet exhaust dissipation. $\dot{\mathcal{A}}^{*}=\dot{\mathcal{E}}_{m}+\dot{\mathcal{E}}_{t h}+\dot{\mathcal{A}}_{t o t}$ is the sum of the anergy that has been generated within the control volume and of the outflows that are going to be dissipated, which is constant when the downstream plane is moved away from the body. For practical applications, $\dot{\mathcal{E}}_{\text {prop }}$ is expected to have several times the magnitude of $\dot{\mathcal{E}}_{q}$.

For low-subsonic flow regimes, typically for $M_{\infty}<0.3$, we can consider the flow incompressible. As a consequence, the mechanical and thermal aspects of the flow can be considered, to the leading order, only weakly coupled. Hence, we can isolate all terms of mechanical (resp. thermal) nature from Eq. (??) to form a mechanical (resp. thermal) exergy relation:

$$
\begin{gathered}
\dot{\mathcal{E}}_{\text {prop }, m}=W \dot{\Gamma}+\dot{\mathcal{E}}_{m}+\dot{E}_{\phi} \\
\dot{\mathcal{E}}_{\text {prop }, t h}+\dot{\mathcal{E}}_{q}+\dot{\mathcal{E}}_{\phi}=\dot{\mathcal{E}}_{t h}+\dot{\mathcal{A}}_{\nabla T}
\end{gathered}
$$

The important point is that Eq. (??) is actually equal to Eq. (37) of Drela's formulation [? ] if we consider incompressible flows, ignore unsteadiness and assume the outer boundary to reduce to a Trefftz plane. As expected, the exergy approach provides the capability of thermal energy management on top of mechanical energy considerations via Eq. (??).

\section{Far-field Asymptotic Considerations}

It is relevant to study asymptotic developments of the various terms involved in the present formulation when the downstream surface is extended at infinity downstream. We use the superscript ${ }^{\infty}$ to indicate the value of an integral over such a surface:

$$
\dot{\mathcal{A}}^{\infty}:=\lim _{\mathcal{S}_{O} \rightarrow \mathcal{S}_{\infty}} T_{\infty} \int_{\mathcal{S}_{O}} \rho \delta \mathbf{s}(\mathbf{V} \cdot \mathbf{n}) \mathrm{d} \mathcal{S}
$$

The terms we are interested in are those of Eq. (??) for the case of an adiabatic surface:

$$
\dot{\mathcal{E}}_{\text {prop }}=W \dot{\Gamma}+\dot{\mathcal{E}}_{m}+\dot{\mathcal{E}}_{t h}+\dot{\mathcal{A}}_{\text {tot }}
$$


The behavior of the right-hand side terms of Eq. (??) will be examined keeping in mind that, as the outer boundary is moved downstream of the airplane, $W \dot{\Gamma}$ remains constant. The linearization of Gibbs equation around $\left(p_{\infty}, T_{\infty}\right)$ proves the thermal exergy to vanish downstream of the configuration, see appendix C. Also, to satisfy the second law of thermodynamics, the outflows of mechanical exergy have to decrease towards zero, being dissipated by viscous dissipation. Inserting these considerations into Eq. (??) yields:

$$
\dot{\mathcal{E}}_{\text {prop }}-\dot{\mathcal{A}}_{\text {tot }}^{\infty}=W \dot{\Gamma}
$$

As a result, one can briefly consider three different cases:

a. $\dot{\mathcal{E}}_{\text {prop }}=\dot{\mathcal{A}}_{\text {tot }}^{\infty}$, which means that all work potential (initially stored in the fuel) has been destroyed. $W \dot{\Gamma}=$ 0 : the aircraft is in mechanical equilibrium.

b. $\dot{\mathcal{E}}_{\text {prop }}>\dot{\mathcal{A}}_{\text {tot }}^{\infty}$, which means that the propulsion system has provided more exergy than required for equilibrium flight. $W \dot{\Gamma}>0$ : the aircraft would gain energy height and store the exergy supplied in excess as mechanical energy.

c. $\dot{\mathcal{E}}_{\text {prop }}<\dot{\mathcal{A}}_{\text {tot }}^{\infty}$, which means that the exergy supplied by the propulsion system is not able to balance the total exergy destruction involved. $W \dot{\Gamma}<0$ : the aircraft would lose energy height and become a power source in descent [?].

In agreement with [? ? ], the aircraft acts as an accumulator of mechanical energy that is released during descent. When in steady flight, the exergy of the system tends to zero and therefore the system tends to thermodynamic equilibrium, i.e. thermal and mechanical equilibriums. Locally, the temperature, pressure and velocity tend towards their reference values, the system gets in equilibrium with its surroundings.

\section{Aerodynamic Flows over Unpowered Configurations}

Although the formulation is primarily developed for powered configurations, its restriction to unpowered airframe cases is presented along with the description of the flow from an exergy standpoint. Then, connections are made to some well-known far-field drag expressions to demonstrate that their underlying theory can be related to exergy considerations. 


\section{A. Restricted Exergy-based Formulation}

In the absence of propulsion systems, $\dot{\mathcal{E}}_{\text {prop }}=0$, and considering adiabatic surfaces, $\dot{\mathcal{E}}_{q}=0$. As no thrust is being generated, from Eq. (??) in appendix A, we get $W \dot{\Gamma}=-D V_{\infty}$, which means that Eq. (??) simply reads:

$$
D V_{\infty}=\dot{\mathcal{E}}_{m}+\dot{\mathcal{E}}_{t h}+\dot{\mathcal{A}}_{\phi}+\dot{\mathcal{A}}_{\nabla T}+\dot{\mathcal{A}}_{w}
$$

which can be considered as an extension to transonic flows of the exergy-based approach to incompressible flows of Li et al. [? ? ].

Physically speaking, if we consider a fixed body with an incoming flow as in wind tunnel testing, the following exergy-based interpretation can be suggested. The incoming flow has a certain exergy due to its velocity with reference to the fixed body reference frame. As the flow passes in the vicinity of the obstacle, boundary layers develop and slow the fluid down. This reduction in kinetic energy means that a portion of the exergy of the flow is destroyed. The total enthalpy massflow across the outer boundary is zero [? ], meaning that the total energy is effectively conserved. However, there has been a change in forms: viscous dissipation has converted kinetic energy into thermal energy at temperature $T$ whose field is then made uniform by thermal mixing.

Inserting the internal energy relation (??) into the thermal exergy outflow definition Eq. (??) and invoking the divergence theorem, one gets that thermal exergy outflow can be decomposed into:

$$
\dot{\mathcal{E}}_{t h}=\dot{\mathcal{E}}_{\phi}-\dot{\mathcal{A}}_{\nabla T}-\int_{\mathcal{V}}\left(p-p_{\infty}\right) \nabla \cdot \mathbf{V} \mathrm{d} \mathcal{V}
$$

The viscous dissipation is a source of thermal exergy resulting from the conversion of kinetic energy into thermal energy while the thermal anergy destroys this work potential. The last term being associated with compressibility, the duality of what is named thermal exergy is highlighted once again. Inserting Eq. (??) into Eq. (??), on gets that, except for the term associated to the shock waves which differs slightly[? ], Eq. (??) is equal to the restriction to unpowered configurations of the mechanical energy balance proposed by Drela [? ] if we consider steady mean flows. This author also demonstrated that, outside of the viscous wakes and propulsion plumes, the total mechanical energy $\dot{E}_{u}+\dot{E}_{v}+\dot{E}_{p}$ represents what is generally referred to as the drag power of the lift-induced vortices, in agreement with various previous approaches [? ? ]. 


\section{B. Connection to Far-field Drag Approaches}

In 1925, Betz proposed to evaluate the impact on the flow of the body with a focus on experimental wake survey [?] which led to a great variety of far-field drag expressions since then. As a consequence, the present section does not pretend to be an extensive review of all available expressions but rather aims at making connections between the proposed exergy-based relation (??) and some of the most popular/recent formulations.

\section{Wake-integral Formulations}

In 1999, van Dam gave a review of existing methodologies with a focus on computational fluid dynamics experiences [? ]. Building on the original work of many contributors, it was first assumed that the viscous stresses can be dropped from Eq. (??) and variations of total enthalpy neglected. Then, introducing the Gibbs equation and neglecting small interaction drag as well as density fluctuations, the following popular drag expression was proposed:

$$
D=\int_{\mathcal{T P}} p_{\infty} \frac{\delta \mathrm{s}}{R} \mathrm{~d} \mathcal{S}+\int_{\mathcal{T} \mathcal{P}} \frac{1}{2} \rho_{\infty}(\psi \xi) \mathrm{d} \mathcal{S}
$$

where $\psi$ and $\xi$ are respectively the crossflow stream function and the streamwise or trailing vorticity. This expression was proposed by van der Vooren and Slooff [? ] who also suggested the use Eq. (??) to distinguish between boundary layer drag and wave drag. According to Giles and Cummings [? ], this equation can equivalently be written as follows:

$$
D=\int_{\mathcal{T} \mathcal{P}} p_{\infty} \frac{\delta \mathrm{s}}{R} \mathrm{~d} \mathcal{S}+\int_{\mathcal{T} \mathcal{P}} \frac{1}{2} \rho_{\infty}\left(v^{2}+w^{2}\right) \mathrm{d} \mathcal{S}
$$

As the vortex drag term decreases, there is a corresponding increase in the entropy term, because the total drag must remain constant. Note however that application of this expression is actually tricky for numerical flows because, due to numerical dissipation, the accurate computation of the kinetic energy term can only be achieved close to the body [? ? ] so that the downstream plane should be located nearby the solid. This may violate the assumptions made for the derivation, mainly that density and axial velocity fluctuations may not be negligible. As a consequence, whatever the location of the downstream plane, there is an inherent imprecision in applying Eq. (??) for numerical far-field drag prediction.

This approach, which still forms the underlying theory of many current formulations for the far-field 
drag prediction and decomposition [? $\quad$ ? $\quad$ ? $\quad$ ? $\quad$ ? $\quad$ ? ], can actually be considered as a simplification of the exergy-based relation (??). This statement is proved by simply multiplying Eq. (??) by the freestream velocity $V_{\infty}$ and by replacing $p_{\infty}$ by $\rho_{\infty} R T_{\infty}$ to get:

$$
D V_{\infty}=T_{\infty} \int_{\mathcal{T P}} \rho_{\infty} \delta \mathrm{s} V_{\infty} \mathrm{d} \mathcal{S}+\int_{\mathcal{T P}} \frac{1}{2} \rho_{\infty}\left(v^{2}+w^{2}\right) V_{\infty} \mathrm{d} \mathcal{S}
$$

If we consider the outer boundary $\mathcal{S}_{O}$ to reduce to a Trefftz plane, on which $(\mathbf{V} \cdot \mathbf{n}) \mathrm{d} \mathcal{S}=\left(V_{\infty}+u\right) \mathrm{d} \mathcal{S} \simeq$ $V_{\infty} \mathrm{d} \mathcal{S}$, Eq. (??) reduces to relation (??) if $\rho \simeq \rho_{\infty}$ and if $\dot{E}_{u}, \dot{E}_{p}$, and $\dot{\mathcal{E}}_{t h}$ are neglected. In comparison, the derivation of relation (??) has not required any assumption[? ] about the flow, and, as a consequence, is expected to have a greater level of accuracy.

\section{Oswatitsch's Formulation}

In 1956, Oswatitsch came to a result generally referred to as entropy drag [? ]. Choosing the control surface in the far field of the body, he neglected second order terms in the drag expression Eq. (??) and showed that:

$$
D V_{\infty}=\lim _{\mathcal{S}_{O} \rightarrow \mathcal{S}_{\infty}} \int_{\mathcal{S}_{O}} \rho\left(T_{\infty} \delta \mathbf{s}-\delta h_{i}\right)(\mathbf{V} \cdot \mathbf{n}) \mathrm{d} \mathcal{S}
$$

which he further simplified by neglecting the change in total enthalpy:

$$
D V_{\infty}=\lim _{\mathcal{S}_{O} \rightarrow \mathcal{S}_{\infty}} T_{\infty} \int_{\mathcal{S}_{O}} \rho \delta \mathbf{s}(\mathbf{V} \cdot \mathbf{n}) \mathrm{d} \mathcal{S}
$$

This last equation is well-known by drag prediction specialists as being capable of determining the rate of work of total drag experienced by a body that does not exchange energy (neither mechanical nor thermal) with the flowing fluid. The outer boundary of the control volume must enclose all entropy changes caused by the body in the flow field that in some cases extend far away from the body, for example, those caused by the decay of the vortex sheet behind a wing. As the integral converges towards its final value, the overall impact of the body on the flow is considered and from an energy standpoint, this illustrates the fact that the system tends towards a thermodynamic equilibrium, which is characterized by a maximum entropy.

The aerodynamic conditions of Oswatitsch development are similar to those assumed for the asymptotic considerations introduced in paragraph ?? so that the same result is to be found: Eq. (??) precisely reduces to Eq. (??). Oswatitsch's formulation is an asymptotic development which makes it rather difficult to apply 
to realistic CFD applications due to constraints on mesh extent. In comparison, Eq. (??) has the advantage of being valid close to the body under study.

Noteworthy, Oswatitsch additionally made the connection to a theorem of thermodynamics according to which the decrease of the useful work of a heat engine (as compared with the theoretical efficiency) is equal to the entropy increase of the total system multiplied by the ambient temperature: the Gouy-Stodola Theorem [? ]. As a consequence, and by virtue of Eq. (??), Oswatitsch can be considered as having provided the first exergy-based formulation for the prediction of airframe aerodynamic performance [? ? ? ].

Although the present selection is not exhaustive, it has been shown that many (past and current) momentum-based far-field drag expressions required some thermodynamic considerations and that their underlying theory can be consistently related to the proposed exergy-based formulation.

\section{Discussion on the Formulation}

\section{A. Design Perspective}

Designers being used to manipulate non-dimensionalized values to compare different configurations, in a direct analogy to traditional drag coefficient, all power terms of relation (??) can be non-dimensionalized by the power of the freestream dynamic pressure-force in order to express power coefficients. For example, for $\dot{\mathcal{E}}_{\text {prop }}$ :

$$
C \dot{\mathcal{E}}_{\text {prop }}=\frac{\dot{\mathcal{E}}_{\text {prop }}}{0.5 \rho_{\infty} V_{\infty}^{3} A_{\text {ref }}}
$$

By doing so, the magnitude of the power coefficients is directly comparable to the traditional drag coefficients. Also, the absence of thrust and drag could appear somewhat puzzling in a design perspective. However, the knowledge of these two forces is not mandatory for estimating basic performance of the configuration under study. For example, as for the power balance method [? ], an exergy-based range equation can be introduced, see appendix D:

$$
\mathcal{R}_{\varepsilon}=\frac{1}{E S F C}\left(\frac{C L}{C \dot{\mathcal{A}}^{*}}\right) \ln \left(\frac{W_{i}}{W_{f}}\right)
$$

where, for propulsion, ESFC is the exergy specific fuel consumption as defined by Eq. (??). For aerodynamics, $C L$ is the (traditional) lift coefficient and $C \dot{\mathcal{A}}^{*}$ is the power coefficient of the sum of all outflows 
and anergy generation within the control volume. For structures, it is the (usual) ratio of the initial to final weight. As expected, reducing the specific exergy consumption as well as all exergy outflow and destruction would improve the performance of the vehicle.

Additional developments are likely to be required before the approach can be readily adopted by aircraft designers, however, chances are good that it would fit traditional aircraft performance indicators.

\section{B. Original Contributions and Relations to Existing Work}

The present formulation is a continuation of the various recent efforts mentioned in the introduction, especially those of Riggins et al. [? ? ? ] focusing on hypersonic vehicles with simplified model examples. On the contrary, the focal point of the present formulation is commercial aircraft flying at subsonic to transonic regimes with an emphasis on high-fidelity CFD applications [? ? ]. Also, the present formulation provides a richer phenomenological decomposition of the outflows which is analogous, for the mechanical part, to the one proposed by Drela [? ].

Over this mechanical energy balance, the formulation proposed here has the advantage of being a component of a larger and well-founded vehicle design philosophy based on exergy management [? ? ? ] for tracking and estimating any potentially recoverable waste and linked to thermoeconomic considerations [? ? ]. Additional benefit comes from the thermal management capability which could be applied, for example, to efficiently integrate heat exchangers on aircraft.

Regarding propulsion, a large panel of highly integrated propulsion devices can be evaluated and efficiency characterization can be achieved with thermal energy considerations and for under-/over-expanded nozzles. In the case of more traditional propulsion systems integration, the propulsive exergy term can be decomposed to express the propulsive thrust power and therefore the approach proposed here could fit conventional drag/thrust bookkeepings [? ? ]. Also, greater insight into jet engines' performance could be achieved through the use of dedicated figures of merit such as gas horsepower or thrust work potential [? ? ].

As regards unpowered configurations, although not the main focus of the present work, the restricted formulation is well-founded and can be considered as an extension to transonic flows of existing drag formulation based on exergy considerations [? ? ]. Additionally, its derivation requires less assumptions about the 
flow than for many drag prediction formulations based on momentum analysis [? ? ? ? ?

Finally, having a single formulation and numerical (post-processing) code for both engine/airframe integration and aircraft thermal management is believed to provide a rational and consistent framework for the design of advanced architectures. To the authors knowledge, no formulation that combines all these various aspects for high-fidelity RANS computations post-processing is currently available to the aerospace community.

\section{Conclusions}

An exergy-based formulation has been derived combining a momentum balance along with a first and second laws analysis. The choice of exergy is motivated by its ability to provide a consistent system-level framework to design complex aerospace systems. The output of the derivation is a balance between the exergy supplied by the propulsion system and its (partial) destruction within the control volume. Relation is made between the aircraft mechanical equilibrium and the flow phenomena that directly influence flight power requirements. As the formulation does not rely on the separate definitions of thrust and drag, it is suitable for the performance evaluation of aircraft concepts with boundary layer ingestion. The common currency aspect of the exergy analysis could facilitate the development of such concepts that are highly multidisciplinary.

Characterization of the various outflows that could be valued was made, i.e. the wakes and jets as well as lift-induced vortices. It was pointed out that, through the exergy point of view, any flow perturbation is seen as a potential for improvement. This represents a radically different perception of the flow as compared to a traditional momentum analysis. Asymptotic considerations made clear that the thermal and mechanical exergy outflows vanish far downstream of the configuration; the work potential being destroyed by viscous dissipation and thermal mixing. When the aircraft is in mechanical equilibrium, all the fuel work potential is destroyed by the various irreversible flow phenomena, while it stores any exergy supplied in excess when more thrust is generated than drag.

Finally, restriction of the formulation to unpowered airframe cases was made and connections to recent/popular drag prediction formulations were provided. It was demonstrated that the underlying theory of many momentum-based far-field drag expressions can be related to exergy considerations, their derivations 
requiring more assumptions about the flow.

\section{Appendices}

\section{A. Aircraft Force Balance}

A force balance of a flying aircraft is reminded for taking into account the mechanical equilibrium of the airplane. This force balance is made in the Earth reference frame in which we consider the aircraft as a mass moving at velocity $V_{\infty}$. We assume that the thrust and the drag act in the flight path direction; the net resultant axial force being noted $F_{x}$. A popular result is that a general force balance [?] yields:

$$
F_{x} V_{\infty}=W \dot{\Gamma}
$$

where we consider the aircraft weight $W$ to be constant. As a consequence, if more thrust is provided than drag, we have that $F_{x} V_{\infty}>0$. This excess power represents a potential for a gain in aircraft mechanical energy, i.e. an increase in height and/or velocity. If $F_{x}=0$, the propulsion system provides as much thrust as the drag of the configuration and the aircraft is in mechanical equilibrium[? ], $W \dot{\Gamma}=0$ and there is no potential for acceleration.

\section{B. Thermal Exergy Breakdown}

From Gibbs equation, Eq. (??), for a perfect gas $(p / \rho=R T)$, one can show that entropy, and therefore anergy, is a function of two independent variables such as temperature and density:

$$
\nabla \mathrm{s}=c_{v} \frac{\nabla T}{T}+R \rho \nabla \frac{1}{\rho}
$$

The integration of this relation between the local state $(T, \rho)$ and the reference conditions $\left(T_{\infty}, \rho_{\infty}\right)$ yields:

$$
\delta \mathrm{s}(T, \rho)=\delta \mathrm{s}\left(T, \rho_{\infty}\right)+\delta \mathrm{s}\left(T_{\infty}, \rho\right):=c_{v} \ln \left(\frac{T}{T_{\infty}}\right)+R \ln \left(\frac{\rho_{\infty}}{\rho}\right)
$$

It is thus possible to isolate the anergy variation due to pure thermal processes and due to compressibility effects. This decomposition can be introduced into the thermal exergy definition, Eq. (??), to get:

$$
\dot{\mathcal{E}}_{t h}=\underbrace{\int_{\mathcal{S}_{O}} \rho\left[\delta e-T_{\infty} \delta \mathbf{s}\left(T, \rho_{\infty}\right)\right](\mathbf{V} \cdot \mathbf{n}) \mathrm{d} \mathcal{S}}_{\dot{\mathcal{E}}_{t h(T)}}+\underbrace{\int_{\mathcal{S}_{O}}\left[p_{\infty}-T_{\infty} \rho \delta \mathbf{s}\left(T_{\infty}, \rho\right)\right](\mathbf{V} \cdot \mathbf{n}) \mathrm{d} \mathcal{S}}_{\dot{\mathcal{E}}_{t h(\rho)}}
$$

where the two terms are defined hereafter: 
$\dot{\mathcal{E}}_{t h(T)}:$ the outflow rate of thermal exergy at constant volume

$$
\dot{\mathcal{E}}_{t h(T)}=\dot{\mathcal{E}}_{t h}\left(T, \rho_{\infty}\right):=\int_{\mathcal{S}_{O}} \rho c_{v} T\left[1-\frac{T_{\infty}}{T} \ln \left(\frac{T}{T_{\infty}}\right)\right](\mathbf{V} \cdot \mathbf{n}) \mathrm{d} \mathcal{S}
$$

This is the part of thermal exergy that is proportional to $c_{v}$ and thus named thermal exergy at constant volume. When the magnitude of $\delta T$ is small compared to $T_{\infty}$, we can use a second order Taylor series of $\ln \left(T / T_{\infty}\right)=\ln \left(1+\delta T / T_{\infty}\right)$ to prove that the integrand is equal to $\rho c_{v}\left(T-T_{\infty}\right)^{2} /\left(2 T_{\infty}\right)(\mathbf{V} \cdot \mathbf{n})$ and that the convected quantity is positive [? ].

$\dot{\mathcal{E}}_{t h(\rho)}:$ the outflow rate of thermal exergy associated with volume change

$$
\dot{\mathcal{E}}_{t h(\rho)}=\dot{\mathcal{E}}_{t h}\left(T_{\infty}, \rho\right):=\int_{\mathcal{S}_{O}} p_{\infty}\left[1-\frac{\rho}{\rho_{\infty}} \ln \left(\frac{\rho_{\infty}}{\rho}\right)\right](\mathbf{V} \cdot \mathbf{n}) \mathrm{d} \mathcal{S}
$$

The integrand can be re-written as $\rho R T_{\infty}[1 / x+\ln (x)](\mathbf{V} \cdot \mathbf{n})$ where $x=\rho / \rho_{\infty}>0$ to prove that the convected quantity is strictly positive.

Although the compressibility of the flow can be considered of mechanical nature, as thermal energy and compressibility are closely related outside of viscous regions, they are gathered under the terminology thermal. The distinction of the two phenomena could make sense in regions where there are important expansions/compressions such as in engine nozzles.

\section{Thermal Exergy Outflow}

Assuming the fluid locally weakly perturbated in terms of temperature and pressure, we can linearize Eq. (??) around ( $\left.T \simeq T_{\infty}, p \simeq p_{\infty}\right)$ and neglect second order terms to get:

$$
T_{\infty} \delta \mathbf{s}=\delta e+p_{\infty} \delta\left(\frac{1}{\rho}\right)
$$

which, multiplying by the local mass flow $\rho(\mathbf{V} \cdot \mathbf{n})$ and summing over the outer boundary far downstream yields, using the mass conservation Eq. (??):

$$
\lim _{\mathcal{S}_{O} \rightarrow \mathcal{S}_{\infty}} T_{\infty} \int_{\mathcal{S}_{O}} \rho \delta \mathbf{s}(\mathbf{V} \cdot \mathbf{n}) \mathrm{d} \mathcal{S}=\lim _{\mathcal{S}_{O} \rightarrow \mathcal{S}_{\infty}} \int_{\mathcal{S}_{O}} \rho \delta e(\mathbf{V} \cdot \mathbf{n}) \mathrm{d} \mathcal{S}+\lim _{\mathcal{S}_{O} \rightarrow \mathcal{S}_{\infty}} \int_{\mathcal{S}_{O}} p_{\infty}(\mathbf{V} \cdot \mathbf{n}) \mathrm{d} \mathcal{S}
$$

which can be combined with the definition of thermal exergy Eq. (??) to prove that the thermal exergy far downstream of the airplane is zero, $\dot{\mathcal{E}}_{t h}^{\infty}=0$. On the contrary, the linearization of Gibbs relation is not possible when the surface of integration $S_{O}$ is close to the engine exhaust jet in which case the temperature may be far from the reference conditions which precisely indicates that the thermal exergy $\dot{\mathcal{E}}_{t h}$ is substantial. 


\section{Exergy-based Range Equation}

This appendix presents the derivation of the exergy-based range equation which is obtained in a very similar manner as for the power balance method [?]. During the flight, the time rate of change of weight is linked to the time rate of weight of fuel burnt $\dot{W}_{f}$ :

$$
-\frac{d W}{d t}=\dot{W}_{f}
$$

One can define an exergy specific fuel consumption ESFC as:

$$
E S F C:=\frac{\dot{W}_{f}}{\dot{\mathcal{E}}_{\text {prop }}}
$$

and therefore re-write Eq. (??) as:

$$
-\frac{d W}{d t}=E S F C \dot{\mathcal{E}}_{\text {prop }}
$$

Considering the aircraft in a steady level flight, we have that $W=L$ where $L$ is lift. If we consider adiabatic surfaces, according to Eq. (??), we have that $\dot{\mathcal{E}}_{\text {prop }}=\dot{\mathcal{A}}^{*}$ where $\dot{\mathcal{A}}^{*}$ is the sum of the anergy generated and all outflows. Therefore, one can write:

$$
\dot{\mathcal{E}}_{\text {prop }}=\dot{\mathcal{A}}^{*}=\dot{\mathcal{A}}^{*} \frac{W}{L}
$$

and re-write Eq. (??) as:

$$
-\frac{d W}{d t}=\frac{E S F C}{\left(L / \dot{\mathcal{A}}^{*}\right)} W
$$

Assuming $L / \dot{\mathcal{A}}^{*}$ and $E S F C$ to be constant along the flight path, one can write:

$$
-\frac{d W}{W}=\frac{E S F C}{\left(L / \dot{\mathcal{A}}^{*}\right)} d t
$$

and integrate to get a relation of the weight $W$ at a given time $t$ and the initial weight $W_{i}$ :

$$
-\ln \left(\frac{W}{W_{i}}\right)=\frac{E S F C}{\left(L / \dot{\mathcal{A}}^{*}\right)}(t-0)
$$

For the final time $t_{f}$ of the flight we have, introducing the final weight $W_{f}$ :

$$
t_{f}=\frac{1}{E S F C} \frac{L}{\dot{\mathcal{A}}^{*}} \ln \left(\frac{W_{i}}{W_{f}}\right)
$$


Therefore, the (exergy-based) range $\mathcal{R}_{\varepsilon}$ flown at a given flight velocity $V_{\infty}$ during the flight time $t_{f}$ is simply:

$$
\mathcal{R}_{\varepsilon}:=V_{\infty} \times t_{f}=\frac{1}{E S F C} \frac{L V_{\infty}}{\dot{\mathcal{A}}^{*}} \ln \left(\frac{W_{i}}{W_{f}}\right)
$$

which, introducing the non-dimensionalized lift and anergy power coefficients yields:

$$
\mathcal{R}_{\varepsilon}=\frac{1}{E S F C}\left(\frac{C L}{C \dot{\mathcal{A}}^{*}}\right) \ln \left(\frac{W_{i}}{W_{f}}\right)
$$

\section{Acknowledgments}

This work is supported by the French National Research Agency. The authors are greatly indebted to S. Mouton (ONERA) for introducing the concept of exergy for the assessment of aeropropulsive performance. D. Destarac (ONERA) is acknowledged for the review of the article and for fruitful advice regarding numerical aspects and far-field drag prediction. The authors also benefited from suggestions from D. Bailly (ONERA).

\section{References}

[1] Gohardani, A. S., Doulgeris, G., and Singh, R., "Challenges of future aircraft propulsion: A review of distributed propulsion technology and its potential application for the all electric commercial aircraft," Progress In Aerospace Sciences, Vol. 47, 2011, pp. 369-391.

[2] Ashcraft, S. W., Padron, A. S., Pascioni, K. A., Stout, G. W., Jr., and Huff, D. L., "Review of Propulsion Technologies for N+3 Subsonic Vehicle Concepts," NASA TM-2011-217239, 2011.

[3] Kawai, R. T., Friedman, D., and Serrano, L., "Blended Wing Body (BWB) Boundary Layer Ingestion (BLI) Inlet Configuration and System Studies," NASA CR-2006-214534, 2006.

[4] Liu, C., Doulgeris, G., Laskaridis, P., and Singh, R., "Thermal cycle analysis of turboelectric distributed propulsion system with boundary layer ingestion,” Aerospace Science and Technology, Vol. 27, 2013, pp. 163-170.

[5] Felder, J., Kim, H. D., and Brown, G., "Turboelectric Distributed Propulsion Engine Cycle Analysis for HybridWing-Body Aircraft," $47^{\text {th }}$ AIAA Aerospace Sciences Meeting Including The New Horizons Forum and Aerospace Exposition, Orlando, FL, 5-8 January 2009.

[6] Drela, M., "Power Balance in Aerodynamics Flows," AIAA Journal, Vol. 47, No. 7, 2009, pp. 1761-1771. doi: $10.2514 / 1.42409$

[7] Pandya, S. A., Huand, A., Espitia, A., and Uranga, A., “Computational Assessment of the Boundary Layer Ingesting Nacelle Design of the D8 Aircraft," $52^{\text {th }}$ AIAA Aerospace Sciences Meeting, National Harbor, MD, 13-17 January 2014. 
[8] Uranga, A., Drela, M., Greitzer, E. M., Titchener, N. A., Lieu, M. K., Siu, N. M., Huang, A. C., Gatlin, G. M., and Hannon, J. A., "Preliminary Experimental Assessment of the Boundary Layer Ingestion Benefit for the D8 Aircraft,” 52 $2^{\text {th }}$ AIAA Aerospace Sciences Meeting, National Harbor, MD, 13-17 January 2014.

[9] Gambill, J., Wiese, D., Claeys, H., Matulich, D., and Weiss, C., "Integrated Aircraft Thermal Management and Power Generation," SAE Technical Paper 932055, 1993. doi:10.4271/932055

[10] Dahm, W. J. A., Allen, N., Razouk, R. R., and Shyy, W., “Challenges and Opportunities in the Next Two Decades of Aerospace Engineering,” Encyclopedia of Aerospace Engineering [online journal], Wiley Online Library, Chap. 2, 2010, pp. 27-37.

[11] Maser, A. C., Garcia, E., and Mavris, D. N., "Characterization of Thermodynamic Irreversibility for Integrated Propulsion and Thermal Management Systems Design," $50^{\text {th }}$ AIAA Aerospace Sciences Meeting including the New Horizons Forum and Aerospace Exposition, Nashville, TN, 9-12 January 2012.

[12] Felder, J. J., Brown, G. V., Kim, H. D., and Chu, J., “Turboelectric Distributed Propulsion in a Hybrid Wing Body Aircraft," $20^{\text {th }}$ ISABE Conference, Gothenburg, Sweden, 12-16 September, 2011.

[13] Rant, Z., "Exergie, ein neues Wort für «technische Arbeitsfähigkeit»," Forsch. Ing. Wes., Vol. 22, No. 1, 1956, pp. $36-37$.

[14] Keenan, J. H., "Availability and irreversibility in thermodynamics," British Journal of Applied Physics, Vol. 2, No. 7, 1951, pp. 183-192.

[15] Moran, M. J., and Sciubba, E., "Exergy Analysis: Principles and Practice," Journal of Engineering for Gas Turbines and Power, Vol. 116, 1994, pp. 285-290.

[16] Sciubba, E., and Wall, G., "A brief Commented History of Exergy From the Beginnings to 2004," Int. J. of Thermodynamics, Vol. 10, No. 1, 2007, pp. 1-26.

[17] Dincer, I., and Rosen, M. A., Exergy, Energy, Environment and Sustainable Development, Elsevier Science Ltd., 2007, Chaps. 1, 2, 18.

[18] Bejan, A., Advanced Engineering Thermodynamics, $3^{\text {rd }}$ ed., John Wiley \& Sons, Inc., 2006, Chaps. 3, 8, 9, 10.

[19] Pellegrini, L. F., Gandolfi, R., da Silva, G. A. L., and de Oliveira, Jr., S., "Exergy Analysis as a Tool for Decision Making in Aircraft Systems Design," 45 ${ }^{\text {th }}$ AIAA Aerospace Sciences Meeting, Reno, NV, 8-11 January 2007.

[20] de Oliveira, Jr., S., Exergy: Production, Cost and Renewability, $1^{\text {st }}$ ed., Springer-Verlag, London, 2013, Chaps. 1, 8.

[21] Roth B., and Mavris, D., "Minimizing Vehicle Environmental and Economic Cost Via Thermodynamic Work Potential," NSF DMII Grantees Conference, Tampa, FL, 2001.

[22] Moorhouse, D. J., "Proposed System-Level Multidisciplinary Analysis Technique Based on Exergy Methods," Journal of Aircraft, Vol. 40, No. 1, 2003, pp. 11-15. 
[23] Bejan, A., "Fundamentals of exergy analysis, entropy generation minimization, and the generation of flow architecture," Int. J. Energy Res., Vol. 26, 2002, pp. 545-565. doi: 10.1002/er.804

[24] Roth, B. A., and Mavris, D. N., "A Generalized Model for Vehicle Thermodynamic Loss Management and Technology Concept Evaluation," Journal of Aircraft, Vol. 40, No. 1, 2003, pp. 62-69.

[25] Bejan, A., Tsatsaronis, G., and Moran, M., Thermal Design and Optimization, 1996, John Wiley \& Sons, Inc., New York, Chap. 8, pp. 405-462.

[26] Bejan, A., and Siems, D. L., "The need for exergy analysis and thermodynamic optimization in aircraft development," Exergy, an International Journal, Vol. 1, No. 1, 2001, pp. 14-24.

[27] Berg, F. T. N., Balchin, M. J., and Keogh, P. S., “New Principles for Dynamic Aircraft Exergy Mapping,” Journal of Aircraft, Vol. 50, No. 4, 2013, pp. 1088-1098. doi: 10.2514/1.C032040.

[28] Tona, C., Raviolo, P. A., Pellegrini, L. F., and de Oliveira Jr, S., "Exergy and thermoeconomic analysis of a turbofan engine during a typical commercial flight," Energy, Vol. 35, 2010, pp. 952-959. doi: 10.1016/j.energy.2009.06.052

[29] Fernández-Villacé, V., and Paniagua, G., "On the exergetic effectiveness of a combined-cycle engines for high speed propulsion," Energy, Vol. 51, 2013, pp. 382-394. doi: 10.1016/j.energy.2012.11.051

[30] Marley, C. D., and Riggins, D. W., "The Thermodynamics of Exergy Losses and Thrust Production in Gas Turbine Engines," $47^{\text {th }}$ AIAA/ASME/SAE/ASEE Joint Propulsion Conference \& Exhibit, San Diego, CA, 31 July - 03 August 2011.

[31] Clarke, J. M., and Horlock, J. H., "Availability and Propulsion," Journal Mechanical Engineering Science, Vol. 17, No. 4, 1975, pp. 223-232.

[32] Riggins, D. W., Camberos, J., Wolff, M, and Bowcutt, K., "Mission-Integrated Exergy Analysis for Hypersonic Vehicles: Methodology and Application," Journal of Propulsion and Power, Vol. 29, No. 3, 2013, pp. 610-620. doi: 10.2514/1.B34733

[33] Gandolfi, R., Pellegrini, L. F., da Silva, G. A. L., and de Oliveira Jr., S., "Exergy Analysis Applied to a Complete Flight Mission of a Commercial Aircraft," $46^{\text {th }}$ AIAA Aerospace Sciences Meeting and Exhibit, Reno, NV, 7-10 January, 2008.

[34] Gandolfi, R., Pellegrini, and de Oliveaira, Jr., S., "More Electric Aircraft Analysis Using Exergy as a Design Comparison Tool," 48 ${ }^{\text {th }}$ AIAA Aerospace Sciences Meeting, Orlando, FL, 4-7 January 2010.

[35] Etele, J., and Rosen, M. A., "Exergy Losses for Aerospace Engines: Effect of Reference-Environments on Assessment Accuracy," 39 $9^{\text {th }}$ AIAA Aerospace Sciences Meeting \& Exhibit, Reno, NV, 10-13 January 2000.

[36] Roth B., "Aerodynamic Drag Loss Chargeability and Its Implications in the Vehicle Design Process," $1^{\text {st }}$ AIAA Aircraft, Technology, Integration, and Operations Forum, Los Angeles, CA, 2001.

[37] Periannan, V., von Spakovsky, M. R., and Moorhouse, D. J., "Investigation of the effects of various energy and 
exergy-based figures of merit on the optimal design of a high performance aircraft system," ASME 2006 International Mechanical Engineering Congress and Exposition, Paper No. IMECE2006-14186, pp. 337-347, Chicago, IL, 5-10 November 2006. doi: 10.1115/IMECE2006-14186

[38] Riggins, D. W., Moorhouse, D. J., and Camberos, J. A., “Characterization of Aerospace Vehicle Performance and Mission Analysis Using Thermodynamic Availability," Journal of Aircraft, Vol. 47, No. 3, 2010, pp. 904-916. doi: $10.2514 / 1.46420$.

[39] Riggins, D. W., Taylor, T., and Moorhouse, D. J., "Methodology for Performance Analysis of Aerospace Vehicles Using the Laws of Thermodynamics," Journal of Aircraft, Vol. 43, No. 4, 2006, pp. 953-963.

[40] Arntz, A., Atinault, O., Destarac, D., and Merlen, A., "Numerical Airframe Aerodynamic Performance Prediction: An Exergy Point of View," $49^{\text {th }}$ International Symposium of Applied Aerodynamics, AAAF, Lille, France, 24-26 March 2014.

[41] Arntz, A., Atinault, O., Destarac, D., and Merlen, A., "Exergy-based Aircraft Aeropropulsive Performance Assessment: CFD Application to Boundary Layer Ingestion," $32^{\text {nd }}$ AIAA Applied Aerodynamics Conference, AIAA Aviation 2014, Atlanta, GA, 16-20 June 2014.

[42] Blazek, J., Computational Fluid Dynamics: Principles and Applications, Elsevier Sciences Ltd., Oxford, 2001, Chap. 7, pp. 225-265.

[43] van Dam, C. P., "Recent experience with different methods of drag prediction," Progress In Aerospace Sciences, Vol. 35, 1999, pp. 751-798.

[44] Oswatitsch, K., Gas Dynamics, Academic Press Inc., New-York, 1956, Chap. 4, pp. 203-210.

[45] Kundu, A. K., Aircraft Design, Cambridge Aerospace Series, Cambridge University Press, New York, 2010, Chap. 13, pp. 417-463.

[46] Moore, J., and Moore, J. G., "Entropy Production Rates from Viscous Flow Calculations, Part I. A Turbulent Boundary Layer Flow," ASME Paper 83-GT-70, ASME Gas Turbine Conference, Phoenix, AZ, 1983.

[47] Adeyinka, O. B., and Naterer, G. F., "Modeling of Entropy Production in Turbulent Flows," Journal of Fluids Engineering, Vol. 126, 2004, pp. 893-899.

[48] Cengel, Y. A., and Boles, M. A., Thermodynamics: An Engineering Approach, $5^{\text {th }}$ ed., McGraw-Hill Higher Education, Boston, MA, 2006, Chap. 8, pp. 423-485.

[49] Giles, M. B., and Cummings, R. M., "Wake Integration for Three-Dimensional Flowfield Computations: Theoretical Development," Journal of Aircraft, Vol. 36, No. 2, 1999, pp. 357-365.

[50] Borel, L., Thermodynamique et Énergétique, $3^{\text {rd }}$ ed., Vol. 1, Presses Polytechniques et Universitaires Romandes, 1991, Chaps. 1, 10.

[51] Camberos, J. A., and Doty, J. H., "Fundamentals of Exergy Analysis," in Exergy Analysis and Design Optimization 
for Aerospace Vehicles and Systems, edited by Moorhouse, D. J. and Camberos, J. A., Progress in Astronautics and Aeronautics, AIAA, 2011, Chap. 2, pp. 9-76.

[52] Destarac, D., "Far-field / Near-field Drag Balance and Applications of Drag Extraction in CFD," CFD-based Aircraft Drag Prediction and Reduction Lecture Series, Von Karman Institute for Fluid Dynamics, Hampton, VA, 2003.

[53] Smith, L. H., Jr., "Wake Ingestion Propulsion Benefit," Journal of Propulsion and Power, Vol. 9, No. 1, 1993, pp. 73-82. doi:10.2514/3.11487

[54] Kless, J. E., Aftosmis, M. J., Ning, S. A., and Nemec, M., "Inviscid Analysis of Extended-Formation Flight,” AIAA Journal, Vol. 51, No. 7, 2013, pp. 1703-1715. doi: 10.2514/1.J052224

[55] Patterson, Jr., J. C., and Flechner, S. G., "Exploratory Wind-Tunnel Investigation of a Wingtip-Mounted Vortex Turbine for Vortex Energy Recovery," NASA TechnicaI Paper 2468, 1985.

[56] Abeyounis, W. K., Patterson, Jr., J. C., Stough, III, H. P., Wunschel, Lt. Col., A. J., and Curran, P. D., "Wingtip Vortex Turbine Investigation for Vortex Energy Recovery," SAE Aerotech '90, 1990 SAE Aerospace Technology Conference \& Exposition, 1-4 October 1990.

[57] Farokhi, S., Aircraft Propulsion, John Wiley \& Sons, Inc., 2009, Chap. 3, pp. 97-126.

[58] Covert, E. E. (ed.), James, C. R., Kimzey, W. F., Richey, G. K. and Rooney, E. C., Thrust and Drag: Its Prediction and Verification, Progress in Astronautics and Aeronautics, AIAA, 1985.

[59] Riggins, D. W., "Evaluation of Performance Loss Methods for High-Speed Engines and Engine Components," Journal of Propulsion and Power, Vol. 13, No. 2, 1997, pp. 296-304.

[60] Roth, B. A., and Mavris, D. N., "Comparison of Thermodynamic Loss Models Suitable for Gas Turbine Propulsion," Journal of Propulsion and Power, Vol. 17, No. 2, 2001, pp. 324-332.

[61] Riggins, D. W., McClinton, C. R., and Vitt, P. H., "Thrust Losses in Hypersonic Engines Part 1: Methodology," Journal of Propulsion and Power, Vol. 13, No. 2, 1997, pp. 281-287.

[62] Roth, B., "Work Potential Perspective of Engine Component Performance," Journal of Propulsion and Power, Vol. 18, No. 6, 2002, pp. 1183-1190.

[63] Li, H., Stewart, J., and Figliola, R., "Exergy Based Design Methodology for Airfoil Shape Optimization and Wing Analysis," $25^{\text {th }}$ International Congress of the Aeronautical Sciences, ICAS 2006, Hamburg, Germany, 3-8 September 2006.

[64] Monsch, S., Li, H., Harris, R. Stewart, J., Figliola, R., and Camberos, J., "Exergy Based Design Methodology for Wing Shape Optimization and Analysis," 39 $9^{\text {th }}$ AIAA Thermophysics Conference, Miami, FL, 25-28 June 2007. doi: $10.2514 / 6.2007-4053$.

[65] Spalart, P. R., "On the far wake and induced drag of aircraft," J. Fluid Mech., Vol. 603, 2008, pp. 413-430. doi:10.1017/S0022112008001146 
[66] Marongiu, C., Tognaccini, R., and Ueno, M., "Lift and Lift-Induced Drag Computation by Lamb Vector Integration," AIAA Journal, Vol. 51, No. 6, 2013, pp. 1420-1430.

[67] Betz, A., "Ein verfahren zur direkten ermittlung des profilwiderstandes," Zeitschrift für Flugtechnik un Motorluftschiffahrt, Vol. 16, 1925, pp. 42-44.

[68] van der Vooren, J., and Slooff, J. W., "CFD-Based Drag Prediction: State-of-the-Art, Theory, and Prospects," Lectures Notes, AIAA Professional Studies Series, Cours on Drag-Prediction and Measurement, Dutch Aerospace Lab, NLR TP 90247, 1990.

[69] Chao, D. D., and van Dam, C, P., "Wing Drag Prediction and Decomposition," Journal of Aircraft, Vol. 43, No. 1, 2006, pp. 82-90. doi: 10.2514/1.C031766

[70] Ueno, M., Yamamoto, K., Tanaka, K., Murayama, M., and Tognaccini, R., "Far-Field Drag Analysis of NASA Common Research Model Simulation," Journal of Aircraft, Vol. 50, No. 2, 2013, pp. 388-397. doi: 10.2514/1.C031766

[71] Paparone, L., and Tognaccini, R., "Computational Fluid Dynamics-Based Drag Prediction and Decomposition," AIAA Journal, Vol. 41, No. 9, 2003, pp. 1647-1657.

[72] Greitzer, E. M., Bonnefoy, P. A., de la Rosa Blanco, E., Dorbian, C. S., Drela, M., Hall, D. K., Hansman, R. J., Hileman, J. I., Liebeck, R. H., Lovergren, J., Mody, P., Pertuze, J. A., Sato, S., Spakovsky, Z. S., Tan, C. S., Hollman, J. S., Duda, J. E., Fitzgerald, N., Houghton, J., Kerrebrock, J. L., Kiwada, G. F., Kordonowy, D., Parrish, J. C., Tylko, J., Wen, E. A., and Lord, W. K., "N+3 Aircraft Concept Designs and Trade Studies Final Report," Volmue 2: Appendices-Design Methodologies for Aerodynamics, Structures, Weight, and Thermodynamic Cycles, NASA/CR-2010-216794/VOL2, 2010.

[73] Ohta, T., Energy Technology: Sources, Systems and Frontier Conversion, $1^{\text {st }}$ ed., Pergamon Press, 1994, Chap. 3, pp. 89-116.

[74] As viewed in the Earth-fixed reference frame.

[75] Not considering chemical exergy and chemical equilibrium.

[76] $\int_{\mathcal{S}_{A}}(\overline{\bar{\tau}} \cdot \mathbf{V}) \cdot \mathbf{n} \mathrm{d} \mathcal{S}=\int_{\mathcal{S}_{P}}(\overline{\bar{\tau}} \cdot \mathbf{V}) \cdot \mathbf{n} \mathrm{d} \mathcal{S} \simeq 0$ and $\int_{\mathcal{S}_{O}}(\overline{\bar{\tau}} \cdot \mathbf{V}) \cdot \mathbf{n} \mathrm{d} \mathcal{S} \simeq 0$.

[77] The loss is the subsequent viscous anergy generated to dissipate the transverse kinetic energy.

[78] Strictly speaking, the chemical exergy is function of the reference conditions [? ], i.e. those at the altitude of flight.

[79] Drela evaluates the total pressure drop across the surface.

[80] Besides neglecting the viscous stresses and the heat flux on the outer boundary.

[81] We consider that all forces have the same point of application, that point being the aircraft center of gravity, so that there is no torque imbalance. 